\title{
Simultaneous Visible Light Communication and Distance Measurement Based on the Automotive Lighting
}

\author{
Bastien Béchadergue, Luc Chassagne, Member, IEEE, and Hongyu Guan
}

\begin{abstract}
Visible light communication could be an interesting complement to the IEEE $802.11 p$-based vehicle-to-vehicle communication systems that are sensitive to interferences and delays in dense traffic scenarios such as platooning. Visible light could also provide a redundant distance measure that is crucial for path control in this application. In this paper, a system called visible light communication rangefinder and performing simultaneously vehicle-to-vehicle communication and range-finding using the headlamps and taillights is proposed for the first time. By exchanging a clock signal contained in Manchester-encoded signals, both the following and leading vehicles can share information and estimate their inter-distance through phase-shift measurement. The system is first presented theoretically and it is shown in particular that the Doppler effect has no significant impact on both functions. Then, it is modeled and validated using Simulink. Finally, both the range-finding and communication function are validated experimentally. The rangefinding function is functional up to $25 \mathrm{~m}$, and has a resolution of around $24 \mathrm{~cm}$ at $10 \mathrm{~m}$, whereas the communication function provides a 500 kbps link with a BER below $10^{-6}$ up to $30 \mathrm{~m}$.
\end{abstract}

Index Terms-Vehicle-to-vehicle communication, visible light communication, distance measurement, sensors, platooning

\section{INTRODUCTION}

A LTHOUGH fully autonomous cars are already on the roads for tests, the next generations of vehicles on the market will probably be automated for certain driving phases only. Platooning could be one of these phases. In platooning, a leading vehicle (LV) is followed by following vehicles (FV) that automatically adjust their position using, among others, vehicle-to-vehicle (V2V) communication and distance sensors. Started in the 1960s, the research on platooning has gained strong interest over the last decades to increase traffic flow and security [1]. This technique has thus been developed through several projects [2], [3], among which the European initiative SARTRE that achieved, in 2012, a fully autonomous, infrastructure independent, 5 to $10 \mathrm{~m}$ platoon led by a manually driven vehicle [4]. The European Truck Platooning Network is now targeting by 2020 a 0.3 s gap between two trucks in a platoon, which corresponds to $9 \mathrm{~m}$ at $110 \mathrm{~km} / \mathrm{h}[5]$.

In most platooning projects, $\mathrm{V} 2 \mathrm{~V}$ communication is implemented with IEEE $802.11 \mathrm{p}$-based systems, that support

Manuscript received...; revised... (Corresponding author: Hongyu Guan.) The authors are with the Laboratoire d'Ingénierie des Systèmes de Versailles (LISV), UVSQ, Université Paris-Saclay, 10-12 avenue de l'Europe, 78140, Vélizy, France (email: luc.chassagne@uvsq.fr; hongyu.guan@uvsq.fr).

Bastien Béchadergue is also with the Vedecom Institute, 77 rue des Chantiers, 78000, Versailles, France (email: bastien.bechadergue@gmail.com). high data rates over long ranges. IEEE $802.11 \mathrm{p}$ is at the heart of cooperative intelligent transportation systems (C-ITS), which ensure vehicular networking [6] through the exchange of similar types of messages [7]. In particular, cooperative awareness messages (CAM) are periodically sent to update the vehicle status information such as the speed or the position [8], which are critical to the trajectory control. However, the random access protocols defined by IEEE $802.11 \mathrm{p}$ may cause excessive delays imposing greater $\mathrm{V} 2 \mathrm{~V}$ distances to meet safety requirements [9]. Visible light communication (VLC), which allows data transmission using the vehicle lighting, has thus been proposed as a complementary technology [10]. In conjunction with IEEE $802.11 \mathrm{p}$, it could indeed increase the reliability and scalability of the platoon [11], [12]. Although these works are theoretical analysis, prototyping efforts have also been made and outdoor communications over tens of meters using commercial off-the-shelf (COTS) LED headlamps or taillights have been reported [13], [14], [15], [16].

Similarly, several automotive range-finding technologies already exist [17], [18]. Frequency-modulated continuous-wave (FMCW) or ultra-wide band (UWB) time-of-flight (TOF) radars operating at 24 or $79 \mathrm{GHz}$ are already widely used, especially for adaptive cruise control (ACC) [19]. Light-based sensors such as lidar are also progressively being deployed, despite their relatively high cost [20]. Although all these solutions provide long-range and high-resolution distance measurement, they may be rather sensitive to interferences [21], [22], an issue that will keep growing as the number of equipped vehicles will increase. Global positioning systems (GPS) can also be used for such purpose but this approach does not provide enough accuracy and does not work when the satellites are out of sight. Therefore, in [23], we proposed for the first time a complementary range-finding solution, the visible light rangefinder (VLR), using the headlamps and taillights of the vehicles for distance measurement. This concept was then validated experimentally in [24].

If both functions can be implemented separately using the automotive lighting, it could also be interesting to perform them using a single system. Simultaneous communication and range-finding with the same light source has not been extensively explored. In [25], Mizui et al. proposed a technique called boomerang transmission, where the FV first sends to the LV a pseudo-noise (PN) code signal using a laser source. Then the LV multiplies its own information with this PN code and sends the resulting signal to the FV. The FV is then able to retrieve the data using a PN matched filter. This filter is 
also used to stop a TOF counter, started when the PN signal was first sent, so that an inter-vehicle distance estimation can be achieved. This system has been further developed over the years [26], [27], but needs additional light sources, generally lasers, instead of using the headlamps and taillights.

In this paper, our concept of VLR is extended into a visible light communication rangefinder (VLCR) able to support simultaneously data transmission and range-finding. In [23], the FV sends a simple clock to the LV that receives, processes and re-emits it. The FV then estimates the phase-shift introduced by the round-trip TOF to recover the distance. Here, the FV sends a clock through a Manchester-encoded CAM-like data signal. The LV is thus able to decode the data while recovering the underlying clock that it uses then to send its own information. The FV uses the same method and finally compares the retrieved clock with the original one to estimate their phase-shift and thus the V2V distance. The theoretical principles of the VLCR are further explained in Section II, and an analysis of the error sources impacting the distance measurement is carried out in Section III. The impact of the Doppler effect is in particular studied and found to be negligible. Then, in Section IV, Simulink simulations of the whole system show that our VLCR could be able to measure distances up to $30 \mathrm{~m}$ with a resolution of $66 \mathrm{~cm}$, at a refresh rate of $267 \mathrm{~Hz}$, while keeping the VLC bit-error rate (BER) below $10^{-6}$ over $45 \mathrm{~m}$, at a data rate of $500 \mathrm{kbps}$. Finally, in Section V, both the range-finding and communication function are validated experimentally using prototypes based on COTS automotive lights. The range-finding function is functional up to $25 \mathrm{~m}$, and has a resolution of around $24 \mathrm{~cm}$ at $10 \mathrm{~m}$, whereas the communication function provides a $500 \mathrm{kbps}$ link with a BER below $10^{-6}$ up to at least $30 \mathrm{~m}$.

\section{BASIC PRINCIPLES OF THE Visible Light COMMUNICATION RANGEFINDER}

\section{A. General Working Principles}

Our VLCR is an extension of the VLR, previously proposed and studied through simulations in [23]. Therefore, the rangefinding function of the VLCR is based on the very same principle as the VLR. Unlike usual range-finding technologies, the VLR cannot directly use the reflection by the target of a signal it is sending to estimate the distance. In radar or lidar, this echo is indeed strong enough to be detected and processed, mainly because its carrier wave is generated by a coherent radio or laser source. Here, the headlamps produce a polychromatic and non-coherent white light so that a major part of the optical power is diffused and lost after its reflection. To avoid this problem, the VLR is based on an active reflection principle: the target, here the $L V$, receives the light signal transmitted by the system, here the FV, and reconstructs it before re-emitting it. This way, the echo received by the FV can be properly detected and processed. Such a solution may be incompatible with the FMCW principles but is possible to combine with TOF measurement [23], [28].

In TOF measurement, a pulse is sent toward the target, which reflects it back so that the system receives the echo with a delay proportional to the system/target distance. A variation of this method consists in sending a periodic signal at frequency $f_{e}$ and observing its phase-shift $\varphi$ with the echo [29]. The latter technique is particularly suited with active reflection by the target since it only has to re-emit the signal it is receiving while preserving its phase. In any case, with this method, the phase-shift $\varphi$ is related to the light-velocity $c$ and the $\mathrm{V} 2 \mathrm{~V}$ distance $d$ by:

$$
d=\frac{c}{2 f_{e}} \cdot \frac{\varphi}{2 \pi}
$$

In the VLR, the signal sent is thus a periodic signal, which does not allow the transmission of data. However, the measurement of a phase shift between two data signals is possible. The IEEE 802.15.7-2011 standard is the latest VLC standard that fully defines the different modulations that may be used [30]. For outdoor applications, it recommends the on-off keying non-return-to-zero (OOK-NRZ) modulation in conjunction with Manchester encoding. OOK-NRZ is the simplest form of light intensity modulations: a data bit 0 is transmitted by turning off the light source and a data bit 1 is sent by turning it on. Manchester encoding consists, for its part, to encode a data bit 0 into the symbol 01 and a 1 into 10. It is employed to limit the maximum number of consecutive 1 or 0 to two, thus preventing light flicker. It also removes the low frequency components of the data signal. More importantly, Manchester encoding is classically used in digital communications to ease clock recovery by ensuring frequent signal transitions [31]. Therefore, by sending Manchester-encoded data signals with OOK-NRZ, both ends of the VLR could also exchange a clock that is easy to recover. The combination of all these principles - active reflection and phase-shift measurement on the clock recovered from Manchester-encoded data signals - gives Fig. 1, the block diagram of our new VLCR.

The VLCR operates as follows: the FV first encodes the digital data $d_{f v}$ using Manchester coding in order to build the CAM-like binary message $m_{e}$ to transmit. $m_{e}$ is then used to monitor the LED headlamps intensity through OOKNRZ modulation, so that the data bits are sent to the LV at a fixed rate $f_{e}$. After free space propagation, the transmitted signal is received by the LV through a photodiode (PD). This received signal $m_{p^{\prime}}$ is distorted and delayed compared to $m_{e}$ by a time proportional to the $\mathrm{V} 2 \mathrm{~V}$ distance $d . m_{p^{\prime}}$ is first processed in order to reconstruct the message. This processing step produces two output signals $d_{r^{\prime}}$ and $m_{r^{\prime}}$. On the one hand, $d_{r^{\prime}}$ is sent to a first channel that decodes the received data. On the other hand, $m_{r^{\prime}}$ is sent to a second channel, where a clock recovery phase locked-loop (PLL) is used to retrieve the clock $s_{r^{\prime}}$ it contains.

This clock, of frequency $f_{e}$, is then used by the LV to transmit its own message signal $m_{e^{\prime}}$ with the same generation, coding and emission process as previously described. $m_{e^{\prime}}$ is received and processed after free space propagation by the FV, which simultaneously decodes the LV data $d_{l v}$ and retrieves the clock signal $s_{r}$ contained in the reconstructed data signal $m_{r} . s_{r}$ and the initial clock $s_{e}$ have a phaseshift $\varphi$ proportional to the $\mathrm{V} 2 \mathrm{~V}$ distance $d$. Consequently, the FV can use these signals to determine an estimate $\varphi_{m}$ of $\varphi$, 


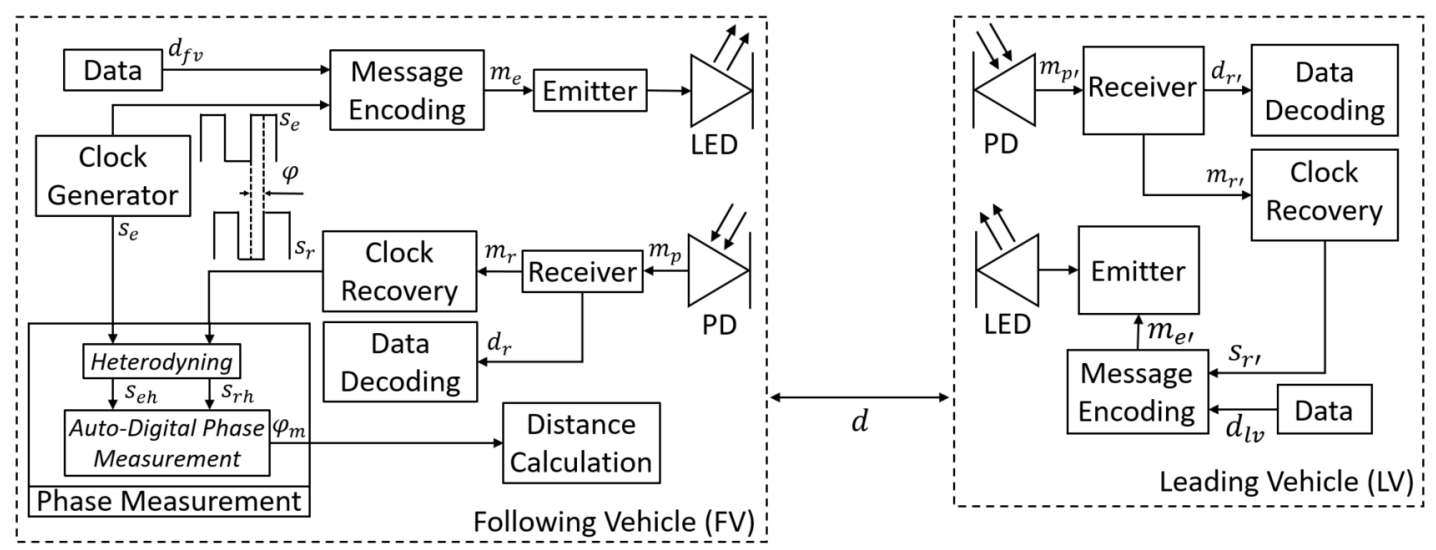

Fig. 1. Block diagram of the VLCR system.

and thus a V2V distance estimate $d_{m}$ using (1). If the main working principles of the VLCR are defined here, the decoding and phase-shift measurement techniques, as well as the signal reconstruction process used on both ends of the system, are still to be detailed. This is the object of the next two sections.

\section{B. VLC Encoding and Decoding Technique}

From the general description of the VLCR working principles just given, we understand that this system is first of all a traditional VLC system. Each vehicle sends a data signal using its headlamps or taillights to the other vehicle which receives it and decodes it. The range-finding function is performed as a parallel task that is not impacting the communication function.

The whole communication chain used by the VLCR from one vehicle to the other is represented on Fig. 2. The data source of the transmitting vehicle, for example its electronic control unit (ECU), generates a data stream that is first encoded using Manchester coding and then reshaped to form data packets of a specific form. In this work, we consider the data packets start with a fixed header followed by the Manchester encoded data payload. The resulting signal, denoted $m_{e}$ when the FV acts as transmitter, is used to drive the transmit LED through an LED driver. After free space propagation, the data light signal is collected by the PD of the receiving vehicle and turned into a voltage signal by a transimpedance amplifier (TIA). This signal is then amplified and bandpass filtered in order to improve its overall SNR so that threshold detection can be used to reconstruct a square data signal $d_{r^{\prime}}$.

(a)

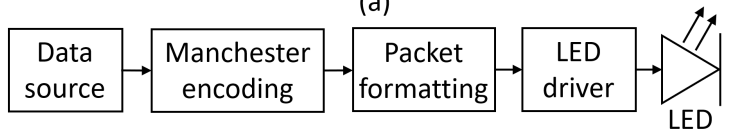

(b)

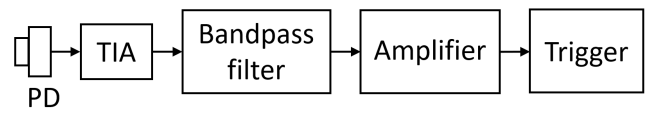

Fig. 2. (a) VLC emission and (b) reception chains.

This reconstructed data signal finally has to be decoded. The method used here consists simply in considering that the current bit state is the value of the data signal $d_{r}$ (or $d_{r^{\prime}}$ ) after each rising edge of a decoding clock of frequency $f_{e}$. To maximize the efficiency of this method, the clock must be synchronized with the data signal, and is thus taken from the clock recovery PLL used for distance measurement. However, the signal processing chain previously described might, as we will see in Section IV-B, introduce distortions on the reconstructed data signal resulting in varying pulse widths. Since these pulse distortions mainly affect the edges of each bit, the recovered clock is here simply delayed by a certain amount of time so that each rising edge occurs approximately at the mid-period of the corresponding bit.

\section{Phase-Shift Measurement Technique}

In parallel with data decoding, the VLCR performs the range-finding function as a two step process. First, the clock is recovered from the signal sent by the FV and received by the LV in order for the LV to send back its own data. Then, the same clock recovery operation is performed by the FV and the resulting signal $s_{r}$ is compared with the initial clock $s_{e}$ sent by the FV in order to extract the phase-shift $\varphi$ between both clocks. This clock recovery process is identical on both ends of the system. The data signal received $m_{p}$ (or $m_{p^{\prime}}$ ) is first reconstructed using the processing chain represented on Fig. 2(b) and the resulting reconstructed signal $m_{r}$ (or $m_{r^{\prime}}$ ) is then fed to the clock recovery PLL to get the clock signal $s_{r}\left(\right.$ or $\left.s_{r^{\prime}}\right)$.

Once both clock signals $s_{e}$ and $s_{r}$ are obtained, the phaseshift measurement process first starts with an heterodyning step: $s_{e}$ and $s_{r}$ are transposed to lower frequency signals $s_{e h}$ and $s_{r h}$. This frequency transposition is performed with a $\mathrm{D}$ flip-flop gate cadenced by a clock $s_{h}$ of frequency $f_{h}$ linked with the frequency of operation $f_{e}$ by a heterodyning factor $r$ such that:

$$
f_{h}=\frac{r}{r+1} f_{e} .
$$

This factor $r$ introduces a small time difference $1 /\left(r f_{e}\right)$ between the periods of the input signal $s_{e}$ or $s_{r}$ and the heterodyning clock $s_{h}$. This way, the shape of the input signal is captured with a time resolution of $1 /\left(r f_{e}\right)$ seconds even though the heterodyning block outputs a value every $1 / f_{h}$ 
seconds in reality. Eventually, such a heterodyning technique is equivalent to a sampler of rate $1 /\left(r f_{e}\right)$ taking $r+1$ periods of input signal to output one full period of heterodyned signal. Therefore, the heterodyned signals $s_{e h}$ and $s_{r h}$ have an intermediate frequency $f_{i}$ given by:

$$
f_{i}=\frac{f_{e}}{r+1} .
$$

This behavior is illustrated by Fig. 3, when $r=10$.

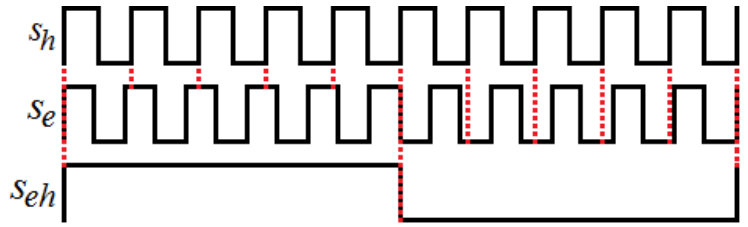

Fig. 3. Heterodyning of a signal $s_{e}$ by undersampling with a synchronized clock $s_{h}$ when $r=10$.

After heterodyning of $s_{e}$ and $s_{r}$, the resulting signals $s_{e h}$ and $s_{r h}$ are compared using a XOR gate to obtain a phase-shift signal $s_{\varphi}$ composed of phase-shift pulses of rate $2 f_{e} /(r+1)$. The width of these pulses is then measured using the autodigital phase-measurement technique [29] illustrated by Fig. 4. The phase-shift pulses $s_{\varphi}$, taken by groups of $N$, are combined with a clock signal $s_{\text {clock }}$ of high-frequency $f_{\text {clock }}$ through an AND gate. The resulting signal $s_{\varphi^{\prime \prime}}$ is composed of several sub-pulses fitting the original phase-shift pulses. The phaseshift $\varphi$ can thus be estimated by simply counting the number of sub-pulses $M$ corresponding to $N$ phase-shift pulses. From this count value, the phase-shift estimate $\varphi_{m}$ can be obtained using:

$$
\varphi_{m}=\frac{2 \pi M f_{e}}{(r+1) N f_{\text {clock }}}
$$

which leads to a distance estimate $d_{m}$ of $d$ given by:

$$
d_{m}=\frac{c}{2} \cdot \frac{M}{(r+1) N f_{\text {clock }}}=\frac{c}{2} \cdot \frac{M f_{i}}{N f_{e} f_{\text {clock }}} .
$$

Note that with this method, the phase-shift is necessarily given modulo- $\pi$, which may introduce an ambiguity in the distance measured. If the true phase-shift $\varphi$ is larger than $\pi$, the system will output a distance corresponding to the difference between this phase-shift and $\pi$. Consequently, there is a nonambiguity range $d_{\text {namb }}$ which corresponds to the distances corresponding to phase shifts between 0 and $\pi$ and which is given by:

$$
d_{n a m b}=\frac{c}{4 f_{e}} .
$$

In any case, the refresh rate of the measurement $f_{\mathrm{VLR}}$ is given by:

$$
f_{\mathrm{VLR}}=\frac{2 f_{e}}{(r+1) N} .
$$

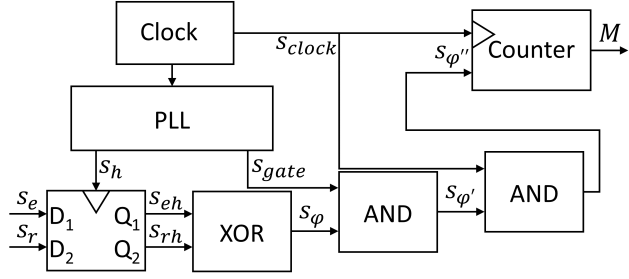

(a)

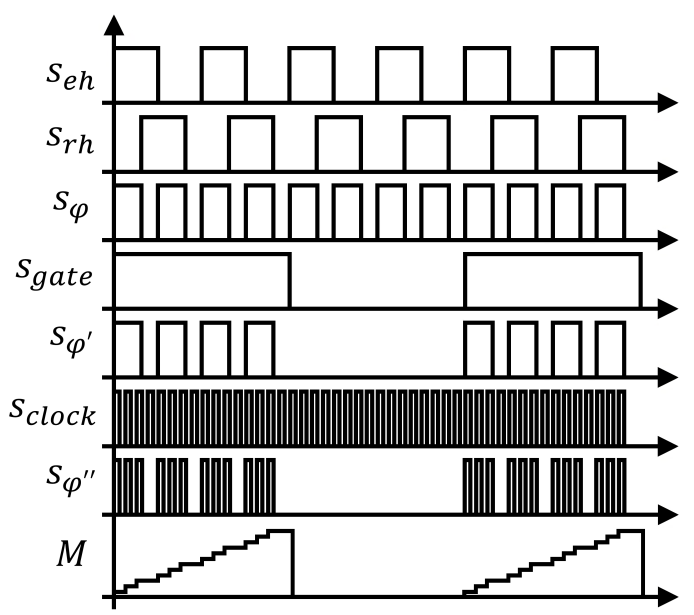

(b)

Fig. 4. (a) Block diagram of the phase-shift measurement system and (b) illustration of its working principles.

\section{Theoretical Analysis of the Sources of ERRORS}

\section{A. General Expression of the Distance Measurement Error}

The distance measurement error $\delta d_{m}$ can be obtained by differentiating the fundamental equation of the VLCR (5), which gives:

$$
\delta d_{m}=d_{m}\left[\frac{\delta M}{M}+\frac{\delta f_{i}}{f_{i}}-\frac{\delta N}{N}-\frac{\delta f_{e}}{f_{e}}-\frac{\delta f_{\text {clock }}}{f_{\text {clock }}}\right] .
$$

Each term has a specific meaning. $\delta f_{\text {clock }}$ and $\delta f_{e}$ represent the frequency drifts of the signals $s_{\text {clock }}$ and $s_{e}$ that may be caused by temperature variations or age. $\delta N$ accounts for the potential non-synchronization between the gate signal $s_{\text {gate }}$ and the phase-shift signal $s_{\varphi}$ whereas $\delta M$ stands for the \pm 1 counting error that may be contained in $M$ [29]. Logically, the latter source of error can be strongly mitigated by increasing the counter clock frequency [23].

Finally, $\delta f_{i}$ gathers three distinct sources of errors. The intermediate frequency $f_{i}$ is indeed the result of a heterodyning process involving the clock signals $s_{h}$ and $s_{r}$. However, $s_{h}$ is derived from the master clock $s_{\text {clock }}$ via a PLL and may thus suffer from frequency drifts that will impact $f_{i}$. On the other hand, $s_{r}$ might have in reality a frequency not equal to but slightly varying around $f_{e}$ because of signal distortions experienced all along the transmission chain. For example, the LED drivers, the headlamps and taillights, the photo-receivers and the signal processing cards have in practice a limited bandwidth whereas the Manchester data signals to transmit $m_{e}$ and $m_{e^{\prime}}$ have by construction a very large spectrum. 
Therefore, their edges will be softened, inducing pulse-width distortions preventing eventually from a perfect recovery of $s_{r}$. In addition, the different processing stages and the clock recovery PLL may add unwanted delays that will change the initial phase of the received clock. The impact of these various sources is surely massive but rather difficult to isolate in a formal way and will thus be studied through simulations in Section IV. However, $\delta f_{i}$ also hides a third source of errors, this time purely intrinsic to the heterodyning step.

\section{B. Heterodyning as a Source of Errors}

In Fig. 3, the heterodyning process is illustrated in the case of an input signal $s_{e}$ that is synchronized with the heterodyning clock $s_{h}$ so that both signals have simultaneous rising edges every $r$ periods. The resulting heterodyned signal $s_{e h}$ is then the perfect transposition of $s_{e}$ at frequency $f_{i}$. Unfortunately, the synchronization, in the sense just defined, of $s_{r}$ with $s_{h}$ is very unlikely since $s_{r}$ contains an uncontrollable phase-shift induced by the return-trip TOF and the various processing delays. The reality will most probably look like the situation represented on Fig. 5.

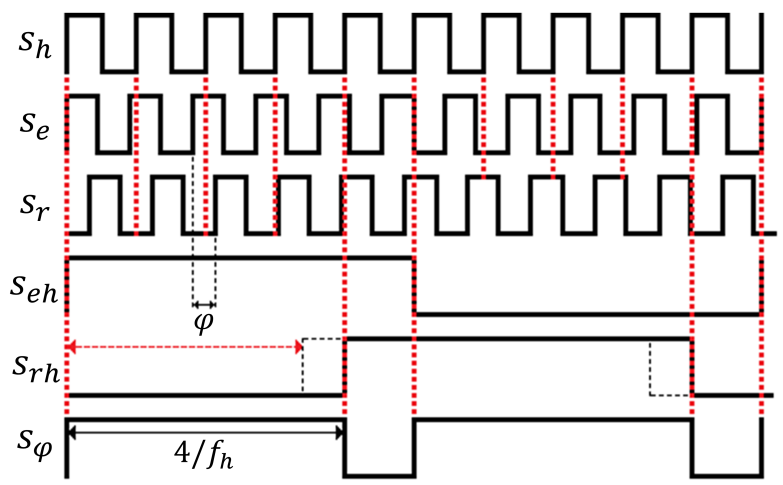

Fig. 5. Illustration of the production of two phase-shift pulses with an heterodyning of factor $r=10$.

In such a case, $s_{h}$ might not detect the state transitions of $s_{r}$. For example, on Fig. 5, we can see that the first rising edge of $s_{h}$ occurs while the input signal $s_{r}$ is at low level, leading logically to a low level of $s_{r h}$. We have then to wait for the fifth rising edge of $s_{h}$ to get the first rising edge of $s_{r h}$ but this transition is clearly occurring shortly after the corresponding edge of $s_{r}$. Therefore, the pulse width of $s_{r h}$, which should follow the black dotted line, has instead a duration equal to an integer multiple of the heterodyning period, here $4 / f_{h}$. In other words, this pulse width contains an error of at most $1 / f_{h}$ seconds, which results eventually in a distance estimate error. In the worst case, there are indeed $\left\lfloor f_{\text {clock }} / f_{h}\right\rfloor$ additional clock counts in the phase measurement. If $f_{\text {clock }}$ is large enough to limit the \pm 1 count-induced measurement error to a few millimeters, the integer part notation can be dropped. By mixing the resulting value with (5), we can show that the maximum distance measurement error $\delta d_{m, h e t}$ induced by the undersampling process is:

$$
\delta d_{m, h e t}=\frac{c}{2 r f_{e}} .
$$

\section{Impact of the Doppler Effect}

The various sources of errors detailed so far are derived considering the $\mathrm{V} 2 \mathrm{~V}$ distance is fixed. However, in real platooning configurations, this distance is always varying which may impact the distance measurement resolution through the well-known Doppler effect. In order to study analytically the potential impact of the Doppler effect, we consider that the FV is approaching the LV from an initial position $x_{0}$ at a constant speed $v_{0}$, as represented on Fig. 6 .

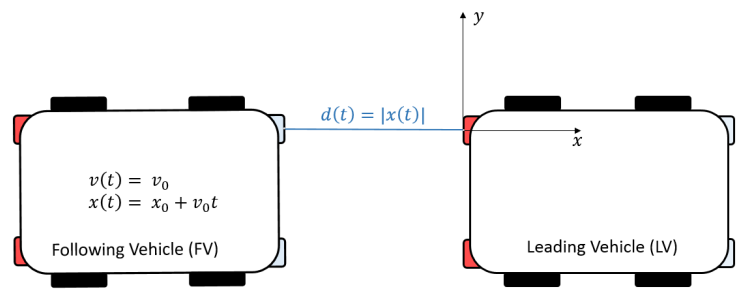

Fig. 6. Geometry of a platoon in a straight line configuration.

We also consider the VLCR is perfect, which means the clock signal retrieved $s_{r}$ is exactly the clock sent $s_{e}$ delayed by the return-trip TOF. In such a case, the only parts of the signals we are interested in are the edges, along with their moment of occurrence and the V2V distance at these moments. Specific notations are used to distinguish these different parameters. For example:

- the $i$-th rising edge of any signal $s_{x}$ will be denoted $s_{x_{i}}$,

- its moment of occurrence will be $t_{x_{i}}$,

- the V2V absolute distance at that moment will be $d\left(t_{x_{i}}\right)$.

Then, if the $i$-th rising edge of $s_{e}$ is sent at time $t_{e_{i}}$, while the $\mathrm{V} 2 \mathrm{~V}$ distance is $d\left(t_{e_{i}}\right)$, its echo will not be received $2 d\left(t_{e_{i}}\right) / c$ seconds later, as in the static case, but with a delay slightly smaller. If $t_{r_{i}}$ is the time of reception and $\tau_{i}=t_{r_{i}}-t_{e_{i}}$ the delay of reception, then:

$$
\tau_{i}=t_{r_{i}}-t_{e_{i}}=-\frac{x_{0}+v_{0} t_{e_{i}}}{c}-\frac{x_{0}+v_{0}\left(t_{e_{i}}-\frac{x_{0}+v_{0} t_{e_{i}}}{c}\right)}{c+v_{0}}
$$

Proof. We know that, in the general case, every rising edge $s_{e_{i}}$, sent by the FV at time $t_{e_{i}}$, will intersect the LV after a delay $\tau_{i, 1}=d\left(t_{e_{i}}\right) / c$. Given the uniform nature of the relative movement between the vehicles, and with respect to the coordinate system defined in Fig. 6, this delay will be:

$$
\tau_{i, 1}=-\frac{x_{0}+v_{0} t_{e_{i}}}{c} .
$$

Assuming the reflection is instantaneous, the echo will then be received by the FV after a second delay $\tau_{i, 2}$ that depends strongly on the speed $v_{0}$. During the return-trip of the signal, if $v_{0}$ is positive, the $\mathrm{FV}$ moves indeed toward the $\mathrm{LV}$, which reduces the $\mathrm{V} 2 \mathrm{~V}$ distance that the echo has to travel. $\tau_{i, 2}$ can thus be simply determined as the intersection between two trajectories: the uniform movement of the echo, which starts from a null initial position with a constant speed $c$ and goes toward the FV, and the uniform movement of the FV, which 
keeps moving toward the $\mathrm{LV}$ at a constant speed $v_{0}$, but now from the initial position $-d\left(t_{e_{i}}+\tau_{i, 1}\right)$ :

$$
-c \tau_{i, 2}=-d\left(t_{e_{i}}+\tau_{i, 1}\right)+v_{0} \tau_{i, 2} .
$$

By solving (12) and introducing (11), we can get:

$$
\tau_{i, 2}=-\frac{x_{0}+v_{0}\left(t_{e_{i}}-\frac{x_{0}+v_{0} t_{e_{i}}}{c}\right)}{c+v_{0}},
$$

which leads to a total delay $\tau_{i}=t_{r_{i}}-t_{e_{i}}=\tau_{i, 1}+\tau_{i, 2}$ of reception of the echo equal to (10).

During this delay, the FV travels a distance $v_{0} \tau_{i}$ which could interact with the distance measurement process, especially the heterodyning step, and thus generate a measurement error. As outlined in Section III-B, the VLCR outputs a measure as soon as an edge of $s_{r}$ is detected by the heterodyning clock $s_{h}$. Let $n$ be the index of the first edge of $s_{r}$ indeed detected by $s_{h}$. According to (10), this edge, first sent at a certain time, will be received with a known delay $\tau_{n}$, which leads to a distance measurement error of the perfect VLCR $\delta d_{m, p e r f}$ equal to:

$$
\delta d_{m, p e r f}=\frac{v_{0} \tau_{n}}{2}+\frac{v_{0} \alpha_{n}}{r f_{e}}+\frac{c \alpha_{n}}{2 r f_{e}},
$$

where $\alpha_{n} \in[0,1[$ is a normalization parameter specific to the $n$-th transmitted pulse.

Proof. We consider here, without loss of generality, the $k$-th phase-shift pulse $s_{\varphi, k}$, delimited by $s_{e h_{k}}$ and $s_{r h_{k}}$, the $k$-th rising edges of $s_{e h}$ and $s_{r h}$, occurring at respective times $t_{e h_{k}}$ and $t_{r h_{k}}$. If we are able to express its width $\Delta t_{k}$, then we can deduce the number of corresponding clock counts $M_{k}$ and thus the distance measured $d_{m_{k}}$ using (5). Then the measurement error $\delta d_{m_{k}}$ will simply be the difference between the distance provided by the VLCR and the true V2V distance when this estimate is output:

$$
\delta d_{m_{k}}=d_{m_{k}}-d\left(t_{r h_{k}}\right) .
$$

A first straightforward expression of $\Delta t_{k}$ is given by:

$$
\Delta t_{k}=t_{r h_{k}}-t_{e h_{k}} .
$$

Considering that $s_{e_{1}}$ and $s_{h_{1}}$, the very first rising edges of $s_{e}$ and $s_{h}$, are occurring simultaneously at time $t=0$, we can deduce, as shown in Fig. 5, that every rising edge of $s_{e h}$ will occur at the same time as a rising edge of $s_{e}$ and $s_{h}$. Therefore, $t_{e h_{k}}$ is an integer multiple of the heterodyning period. In Fig. 5 , we can observe that the end of the first phase-shift pulse occurs five rising edges of $s_{h}, s_{e}$ and $s_{r}$ after its beginning. Back to the general case, let $n$ be this number of rising edges. We can deduce that:

$$
t_{r h_{k}}=t_{e h_{k}}+\frac{n-1}{f_{h}} \Longrightarrow \Delta t_{k}=\frac{n-1}{f_{h}} .
$$

This phase-shift pulse width will correspond to a number of clock counts $M_{k}$ equal to:

$$
M_{k}=\left\lfloor\frac{(n-1) f_{\text {clock }}}{f_{h}}\right\rfloor,
$$

where $\lfloor\cdot\rfloor$ denotes the integer part. This equation highlights the \pm 1 count error pointed out in Section III-A, which does not exceed $1 \mathrm{~mm}$ if the counter frequency is carefully chosen. Therefore, we can drop here the integer part notation and deduce, by mixing (18) with (5), that:

$$
d_{m_{k}} \approx \frac{c(n-1)}{2 r f_{e}} \text {. }
$$

The problem is now to find a literal expression of $n$. With respect to Fig. 5, we understand that $n$ is simply the index such that:

$$
t_{r_{n-1}}>t_{h_{n-1}} \quad \text { and } \quad t_{r_{n}}<t_{h_{n}}
$$

On the one hand, we know that, in the general case, $t_{h_{i}}=$ $(i-1) / f_{h}$. On the other hand, we can say that $t_{r_{i}}$ is equal to the time $t_{e_{i}}=(i-1) / f_{e}$ of emission of the corresponding edge $s_{e_{i}}$ plus the return-trip TOF $\tau_{i}$ given by (10). Considering these expressions of $t_{h_{i}}$ and $t_{r_{i}}$, the two inequalities in (20) lead to:

$$
n \leq r f_{e} \tau_{n}+1
$$

Since $n$ is an integer, then its true value will be $n=$ $\left\lfloor r f_{e} \tau_{n}+1\right\rfloor+1$ or equivalently:

$$
n=r f_{e} \tau_{n}+1+\alpha_{n},
$$

where $\alpha_{n} \in[0,1[$ is a real value such that $n \in \mathbb{N}$. By introducing this expression in (19), we can show that:

$$
d_{m_{k}}=\frac{c \tau_{n}}{2}+\frac{c \alpha_{n}}{2 r f_{e}} .
$$

However, since $\tau_{n}$ is the return trip TOF between the moments $t_{e_{n}}$ and $t_{r_{n}}$, we can deduce that:

$$
\frac{c \tau_{n}}{2}=\frac{d\left(t_{e_{n}}\right)+d\left(t_{r_{n}}\right)}{2},
$$

with:

$$
d\left(t_{e_{n}}\right)=d\left(t_{e h_{k}}\right)-\frac{v_{0}(n-1)}{f_{e}},
$$

and

$$
d\left(t_{r_{n}}\right)=d\left(t_{e h_{k}}\right)-v_{0}\left(\frac{n-1}{f_{e}}+\tau_{n}\right) .
$$

In parallel, using the expression of $t_{r h_{k}}$ given in (17) and mixing it with (22), it can be shown that the absolute V2V distance $d\left(t_{r h_{k}}\right)$ when the VLR outputs the $k$-th distance estimate is:

$$
d\left(t_{r h_{k}}\right)=-x_{0}-v_{0} t_{r h_{k}}=d\left(t_{e h_{k}}\right)-v_{0}(r+1) \tau_{n}-\frac{v_{0} \alpha_{n}}{f_{h}} .
$$

By first mixing (25) and (26) with (24) and introducing the result in (23), an alternate expression of $d_{m_{k}}$ can be found. This expression can then be used with (27) in (15) to get the final expression of the measurement error $\delta d_{m_{k}}$ given in (14).

We can see that the first two terms of this equation are null if the there is no relative movement between the vehicles $\left(v_{0}=0\right)$, whereas the third term is independent of the speed. In addition, all three terms depend on the parameters $\tau_{n}$ or $\alpha_{n}$ which are related to the $n$-th and last transmitted pulse. Therefore, a measure between times $t_{e}$ and $t_{r}$ while traveling at a constant speed $v_{0}$ is the same as the measure started at time $t_{e}$, while the FV is static at a distance $d\left(t_{e_{n}}\right)$, and stopped 
at time $t_{r}$, after the speed has changed abruptly from 0 to $v_{0}$ at time $t_{e_{n}}$ : only the movement between $t_{e_{n}}$ and $t_{r}$ will impact the measurement resolution.

The first term in (14) corresponds to half the distance traveled between the emission at time $t_{e_{n}}$ of the $n$-th pulse and the reception of its echo. It highlights the fact that $\tau_{n}$, which defines the end of the measure, is a delay between two very close but different distances. If for example, $\left|x_{0}\right|=15 \mathrm{~m}$ and $v_{0}=20 \mathrm{~km} / \mathrm{h}$, this term is indeed under $1 \mu \mathrm{m}$ and can thus be neglected. The second term is related to the limited time resolution of the undersampling process already pointed out in Section III-B. It stands for the contribution of the speed $v_{0}$ to this time resolution but only reaches a few tenth of nanometers and can also be neglected so we can conclude that relative V2V movements only induce negligible additional error.

The main source of errors induced by the undersampling step is actually the static error, represented by the third term in (14), and which limit case $\alpha_{n}=1$ is exactly the error highlighted by (9). From (9), we understand that an increase of either the heterodyning factor $r$ or the frequency of operation $f_{e}$ limits this static error. For example, it reaches $9.37 \mathrm{~cm}$ when $f_{e}=1 \mathrm{MHz}$ and $r=1599$ but only $3.75 \mathrm{~cm}$ when $f_{e}=1 \mathrm{MHz}$ and $r=3999$ or $0.94 \mathrm{~cm}$ when $f_{e}=4 \mathrm{MHz}$ and $r=3999$. However, as shown by (7), an increase in the heterodyning factor mechanically reduces the intermediate frequency, and thus the refresh rate $f_{\mathrm{VLR}}$, even though this reduction can be compensated by increasing $f_{e}$. In this work, a frequency of operation $f_{e}=1 \mathrm{MHz}$ is considered. This value is indeed compatible with most COTS automotive lights which have a modulation bandwidth usually limited to a few megahertz. In this case, the non-ambiguity range $d_{\text {namb }}$ given by (6) is $75 \mathrm{~m}$, which is larger than the range we want the VLCR to cover.

\section{Simulation Study of the Visible Light COMMUNICATION RANGEFINDER}

Now that all the different parts of our VLCR have been detailed, we can validate its functioning and evaluate its performances by Simulink simulations. In order to stick to the reality, we have used a standard VLC channel model that is detailed in Section IV-A, and carefully selected the different simulation parameters, as outlined in Section IV-B and IV-C. Therefore, we have been able to evaluate first the communication performances of the VLCR in Section IV-D. Finally, the range-finding capabilities of the system are tested in Section IV-E and compared with those of the VLR alone.

\section{A. The VLCR Channel Model}

The platoon configuration considered in the simulations is represented on Fig. 7. The FV uses one of its headlamps as transmitter and has a PD receiver just under whereas the LV uses a symmetrical equipment on its rear. There are no obstacles between both vehicles and the reflections on the road surface are ignored, which means a continuous direct line-ofsight (LOS) is considered between each transmitter/receiver pairs. In addition, crosstalk is ignored, as well as the Doppler effect.

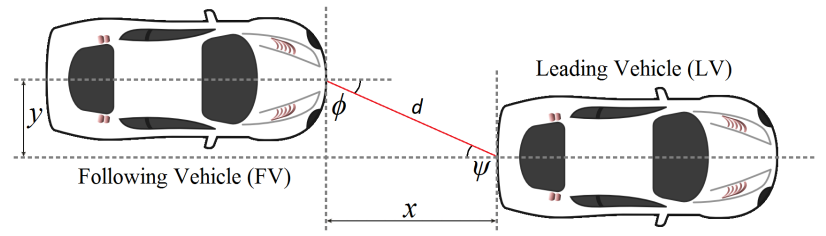

Fig. 7. Platooning configuration considered in the simulations, with $\phi$ and $\psi$ the irradiance and incidence angles and $d$ the $\mathrm{V} 2 \mathrm{~V}$ distance.

The VLC LOS channel is modeled using the classical pathloss additive white Gaussian noise (AWGN) channel model [32]. In this model, the output current $Y(t)$ produced by the PD is linked with the optical power transmitted $X(t)$ by:

$$
Y(t)=\gamma X(t) \otimes h(t)+n(t)
$$

with $\gamma$ the PD responsivity, $h(t)$ the channel impulse response and $n(t)$ the AWGN. The channel frequency response $H(f)$ is usually reduced to its DC gain $H(0)$ because it is considered flat in the frequency range of interest. In addition, the vehicle headlamps and taillights are modeled as Lambertian light sources, although they have in reality complex transmission beam patterns in order to meet international standards. The channel DC gain is then:

$$
H(0)=\frac{(m+1) A_{r}}{2 \pi d^{2}} \cos ^{m} \phi \cos \psi, \quad 0<\psi<\psi_{c},
$$

where $\phi$ and $\psi$ are the irradiance and incidence angles, $A_{r}$ is the radiant sensitive area of the PD, $\psi_{c}$ is the PD field of view and $m$ is the order of Lambertian emission, defined as $m=-\ln 2 / \ln \left(\cos \Phi_{1 / 2}\right)$, with $\Phi_{1 / 2}$ the semi-angle at half power of the LED emitter. If the transmitted optical power is $P_{t}$, then the received light power $P_{r}$ will be $P_{r}=H(0) P_{t}$ and the signal power $S$ at the receiver side will be $S=\gamma^{2} P_{r}^{2}$.

The main noise sources are shot noise and thermal noise. Shot-noise is induced by the incident luminous flux and is mainly due to daylight which is much stronger than any other light source. It is thus modeled as a signal independent white Gaussian noise of variance $\sigma_{\text {shot }}^{2}$ :

$$
\sigma_{\text {shot }}^{2}=2 q \gamma P_{r} B+2 q I_{b g} I_{2} B
$$

with $q$ the electronic charge, $B$ the equivalent noise bandwidth, $I_{b g}$ the background photocurrent, that can be estimated to 5100 $\mu \mathrm{A}$ in case of direct exposition or $740 \mu \mathrm{A}$ in case of indirect exposition [33], and $I_{2}$ a noise bandwidth factor. Thermal noise occurs with charge carriers thermal agitation and is modeled as a signal independent Gaussian noise of variance $\sigma_{\text {thermal }}^{2}$ :

$$
\sigma_{\text {thermal }}^{2}=\frac{8 \pi k T_{K}}{G} \eta A_{r} I_{2} B^{2}+\frac{16 \pi^{2} k T_{K} \Gamma}{g_{m}} \eta^{2} A_{r}^{2} I_{3} B^{3},
$$

with $k$ Boltzmann's constant, $T_{K}$ absolute temperature, $G$ the open-loop voltage gain, $\eta$ the fixed capacitance of photodetector per unit area, $\Gamma$ the field-effect transistor (FET) channel noise factor, $g_{m}$ the FET transconductance and $I_{3}$ a noise-bandwidth factor. The total noise variance $\sigma_{\text {tot }}^{2}$ will 
then be the sum of the shot noise and thermal noise $\sigma_{t o t}^{2}=$ $\sigma_{\text {shot }}^{2}+\sigma_{\text {thermal }}^{2}$, so that the SNR will be:

$$
\mathrm{SNR}=\frac{S}{\sigma_{\text {tot }}^{2}}=\frac{\gamma^{2} H(0)^{2} P_{t}^{2}}{\sigma_{\text {shot }}^{2}+\sigma_{\text {thermal }}^{2}} .
$$

\section{B. Simulink Model of the VLCR}

The software Simulink has been chosen to carry out the simulation study of the VLCR as it provides great flexibility with its block approach. The Simulink model developed is simply the transcription of the block diagram on Fig. 1 with, between the FV and LV main blocks, an additional block for the VLCR bidirectional channel just described.

1) Message Format: In this work, the transmission of CAM-inspired messages is considered. As defined in its standard [8], a CAM is composed of several frames, among which some are compulsory, like speed or heading, whereas others are optional. Therefore, a CAM is a complex message with variable length. However, one could estimate its length is usually between 400 and 600 bytes. Here, the CAM messages are thus modeled by frames of 4000 independent and identically distributed random bits following a uniform distribution, generated by the "Data" blocks of Fig. 1. Then, the "Message Encoding" blocks encode these bits using Manchester coding and incorporate then in frames started by 8 bits synchronization headers $H=00001111$. When the messages are fully encoded, the "Emitter" blocks transmit them bit by bit using OOK-NRZ with a clock of frequency $f_{e}=1 \mathrm{MHz}$, which gives a data rate $R_{b}$ of $500 \mathrm{kbps}$. Note that, as mentioned in Section II-A, Manchester coding limits the low frequency components of the data signal. It actually concentrates most of the signal energy around $f_{e} / 2=500 \mathrm{kHz}$ and distributes the rest in decreasing side lobes. This information is crucial to set up an efficient signal processing strategy.

2) Signal Reconstruction Stages: An identical processing chain is implemented on both ends of the VLCR, in the "Receiver" blocks of Fig. 1. In the context of the VLR, this processing chain was composed of a bandpass filter of bandwidth $100 \mathrm{kHz}$ centered on the frequency of operation $f_{e}=1 \mathrm{MHz}$ and thus able to isolate the fundamental component of the square signal transmitted [24]. Such filtering strategy cannot be used by the VLCR as the signal transmitted is not a square signal anymore but a Manchester encoded data signal with the spectrum just described.

We know from [32] that from a communication perspective, the optimal filtering strategy to apply on Manchester encoded signals consists in a bandpass filter with a low-pass cut-off frequency equal to the data rate $R_{b}$ and a high-pass cutoff frequency equal to $0.01 R_{b}$, that is respectively $500 \mathrm{kHz}$ and $5 \mathrm{kHz}$ here. In practice, these filters are implemented using $2^{\text {nd }}$ order Butterworth analog filters in order to limit the simulation time. This filtering approach will be referred as "VLC filtering" in the rest of the paper. Fig. 8(a) to (d) illustrate the whole processing chain when using such filters. Fig. 8(c) shows the efficient noise removal properties of "VLC filtering" as well as the pulse distortion it introduces. The edges of the signal filtered are indeed strongly softened compared to the original square signal so that the pulse width of the signal reconstructed by zero-crossing detection, shown on Fig. 8(d), may vary for a same logic state.

These pulse width distortions can be mitigated by using an alternative filtering approach which consists in letting some harmonics of the Manchester-encoded signal pass so that the sharpness of its edges is preserved, at the cost of a larger bandwidth and thus of less noise cut. In parallel, removing some of the slowest time-varying components of the signal, contained in the main lobe, will change every rising and falling edges into peaks so that they are easier to detect even though the signal will be weaker. This approach, that will be referred as "Distance Measurement (DM)" filtering in the rest of the paper, is illustrated by Fig. 8(e) to (h). Once again, two $2^{\text {nd }}$ order Butterworth analog filters are employed, but this time with low-pass and high-pass cut-off frequencies $2.5 \mathrm{MHz}$ and $250 \mathrm{kHz}$. Fig. 8(g) shows the ability of these filters to highlight the pulse edges, even when the input is the low SNR signal represented on Fig. 8(f). Symmetrical thresholds can then be used to get the reconstructed message $m_{r}$, as shown in Fig. $8(\mathrm{~h})$.

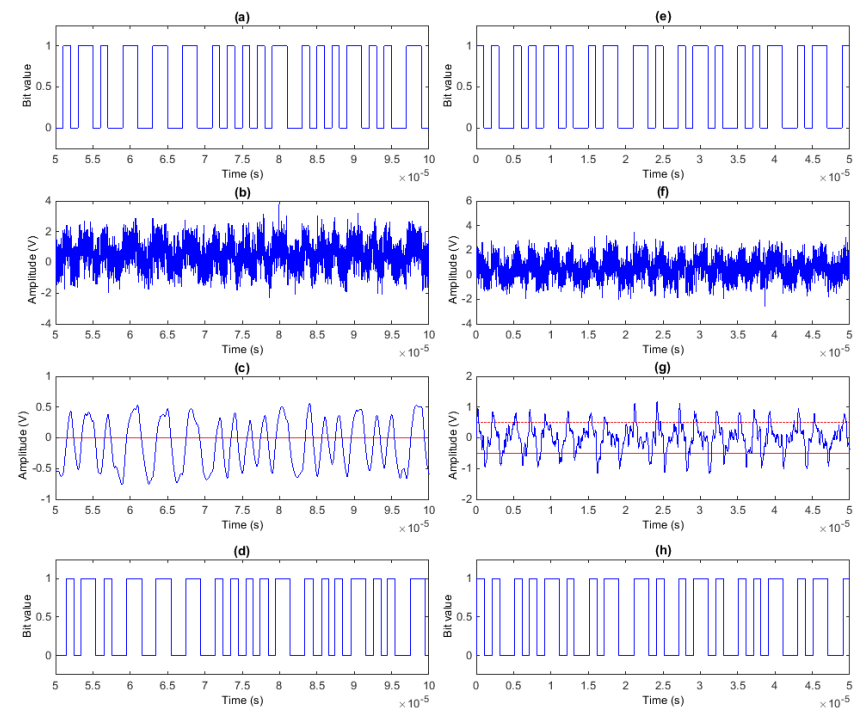

Fig. 8. (a) Data signal transmitted $m_{e^{\prime}}$, (b) signal received $m_{p}$ with AWGN of $\mathrm{SNR}=2$, before processing, and (c) after filtering with $2^{\text {nd }}$ order Butterworth filters of respective low-pass cut-off frequency $500 \mathrm{kHz}$ and high-pass cut-off frequency $5 \mathrm{kHz}$ (blue curve) and triggering threshold (red line), (d) signal $d_{r}$ reconstructed by triggering. (e), (f), (g) and (h) represent the same processing chain with two $2^{n d}$ order Butterworth filters of respective low-pass cut-off frequency $2.5 \mathrm{MHz}$ and high-pass cut-off frequency $250 \mathrm{kHz}$.

The reconstruction properties of these two filtering strategies can be compared by measuring, with a simple cross-correlation analysis, the similarity rate between the original data signal and its reconstructed version. Fig. 9 shows the similarity rates in both cases and compares them with the similarity rate obtained with the narrow bandpass filtering used in [24] for the VLR. It appears clearly that from an SNR of around -5 $\mathrm{dB}$, the similarity rate ensured by "VLR filtering" is larger than with "VLC filtering" thanks to its more accurate edge detection properties. On the contrary, "VLC filtering" is much more reliable at very low SNR because it cuts more noise. This cross-correlation analysis can also be used to show that the 
delay introduced by both approaches is stable and reaches 480 ns with "VLC filtering" and only 90 ns with "DM filtering".

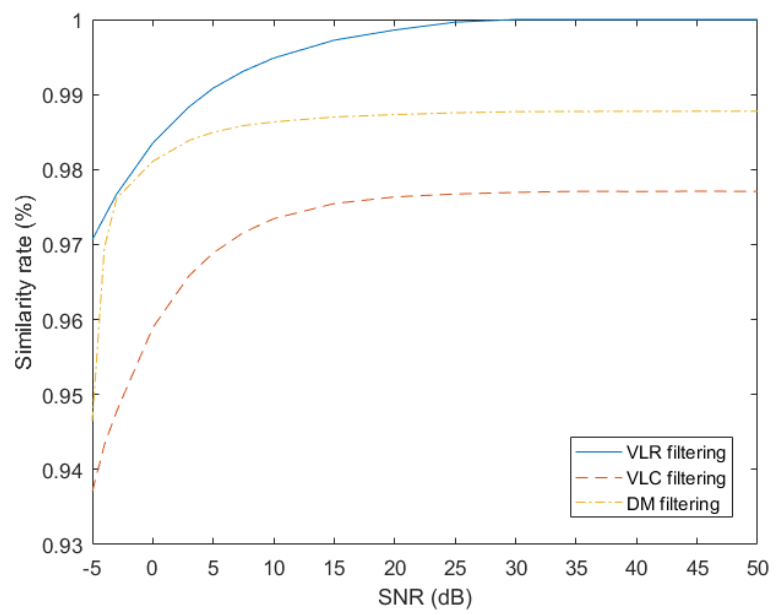

Fig. 9. Evolution of the similarity rate with the SNR according to the filtering strategy used. VLR filtering is given for comparison between the VLCR and the VLR.

3) Clock Recovery PLL: Once the reconstructed signal $m_{r}$ (or $m_{r^{\prime}}$ ) is obtained, a clock recovery PLL is used to get the clock signal $s_{r}$ (or $s_{r^{\prime}}$ ). At this stage, the critical parameter is the time needed by the PLL to converge toward the right phase. In the simulations, this function is modeled using the 'Clock Recovery PLL' block from the 'Mixed-Signal Library' for Simulink, specially designed for this purpose. Therefore, with the help of Manchester coding, it has been observed that when "DM filtering" is used on a message of SNR $=10 \mathrm{~dB}$, the phase of $s_{r}$ converges after less than $2 \mathrm{~ms}$ and remains stable with a rather constant delay of $1 \mathrm{~ns}$ with respect to $m_{r}$. By comparison, a car moving at $130 \mathrm{~km} / \mathrm{h}$ only travels around 7 $\mathrm{cm}$ in $2 \mathrm{~ms}$ so the clock recovery is not a critical step in terms of processing time for dynamic range-finding applications. Note that this $1 \mathrm{~ns}$ delay is partly achieved thanks to the accurate edge detection provided by "DM filtering" since it reaches 250 ns when "VLC filtering" is used. However, note also that this value are given modulo- $2 \pi$, which means that it is hard to know exactly which filtering strategy experiences overall the smallest delay.

\section{Summary of the Simulation Parameters}

The values of the channel parameters and of the intrinsic parameters of the VLCR are listed in Table I. The receiver parameters are realistic values for a PD whereas the channel parameters are taken from [32] and correspond to typical values for a FET-based preamplifier. Since the emission and irradiance angles $\phi$ and $\psi$ are null, the LV and FV both share the same longitudinal axis.

This assumption also allows to bypass the inability of the Lambertian model to capture the complexity of automotive beam patterns. The Lambertian model is indeed fully determined by the semi-angle at half power $\Phi_{1 / 2}$ and the total optical power transmitted $P_{t}$. Since lateral variations between the vehicles are not simulated, these parameters are simply set so that the luminous intensity produced by the light on the lane of the vehicle, just under the horizontal plane of emission, reaches the value defined by automotive lighting regulations UNECE R112 for LED headlamps [34] and UNECE R7 for taillights [35]. This luminous intensity must indeed reach at least $1700 \mathrm{~cd}$ with both headlamps in the first case and at most $730 \mathrm{~cd}$ per taillight in the second case. In addition, note that the limited modulation bandwidth of the headlamps and taillights is modeled on both ends of the VLCR with simple low-pass filters.

Now that our simulation model is fully described, we can review the simulation results.

TABLE I

Simulation MOdel Parameters

\begin{tabular}{|c|c|c|c|}
\hline Parameter & Value & Parameter & Value \\
\hline \multicolumn{2}{|c|}{ General parameters } & \multicolumn{2}{|c|}{ Emitter parameters } \\
\hline$f_{e}$ & $1 \mathrm{MHz}$ & $P_{t}$ & $2 \mathrm{~W}(\mathrm{FV}) / 1 \mathrm{~W}(\mathrm{LV})$ \\
\hline$R_{b}$ & $500 \mathrm{kbps}$ & $\Phi_{1 / 2}$ & $20^{\circ}$ \\
\hline Message length & 4000 bits & \multicolumn{2}{|c|}{ Receiver parameters } \\
\hline Header frame & 00001111 & $\gamma$ & $0.5 \mathrm{~A} / \mathrm{W}$ \\
\hline$f_{\text {clock }}$ & $100 \mathrm{MHz}$ & $A_{r}$ & $50 \mathrm{~mm}^{2}$ \\
\hline$r$ & 1500 & $\psi_{c}$ & $55^{\circ}$ \\
\hline$N$ & 5 & \multicolumn{2}{|c|}{ Channel parameters } \\
\hline$T_{r}$ & $267 \mathrm{~Hz}$ & $\phi$ & $0^{\circ}$ \\
\hline \multicolumn{2}{|c|}{ VLC filtering } & $\psi$ & $0^{\circ}$ \\
\hline Type & Butterworth & $I_{b g}$ & $740 \mu \mathrm{A}$ \\
\hline Order & 2 & $I_{2}$ & 0.562 \\
\hline$f_{-3 d B(L P)}$ & $500 \mathrm{kHz}$ & $B$ & $5 \mathrm{MHz}$ \\
\hline$f_{-3 d B(H P)}$ & $5 \mathrm{kHz}$ & $T_{K}$ & $298 \mathrm{~K}$ \\
\hline \multicolumn{2}{|c|}{$D M$ filtering } & $\eta$ & $112 \mathrm{pF} / \mathrm{cm}^{2}$ \\
\hline Type & Butterworth & $G$ & 10 \\
\hline Order & 2 & $\Gamma$ & 1.5 \\
\hline$f_{-3 d B(L P)}$ & $2.5 \mathrm{MHz}$ & $g_{m}$ & $30 \mathrm{mS}$ \\
\hline$f_{-3 d B(H P)}$ & $250 \mathrm{kHz}$ & $I_{3}$ & 0.0868 \\
\hline
\end{tabular}

\section{VLC Performances}

The communication function is first tested by evaluation of the BER and packet-error rate (PER) evolutions with the V2V distance $d$. The following results are obtained by transmitting $10^{6}$ bits divided into 250 packets, separated by synchronization frames. The BER is measured as the ratio of wrong bits over the total number of bits transmitted and is obtained by comparing the Manchester-encoded bits transmitted and decoded. Similarly, the PER is the ratio of lost packets over the total number of packets, knowing that a packet is considered lost if at least one bit in the packet is wrong. Fig. 10 shows the BER and PER evolution with $d$ when either "VLC filtering" or "DM filtering" is used.

Several conclusions can be drawn. First of all, it appears clearly that the curves with "VLC filtering" are shifted versions of those obtained with "DM filtering". In other words, the behavior of both filtering approaches is similar once the first errors appear, but the range from which these errors start to appear is larger with "VLC filtering". This result can be explained by the fact that "DM filtering" has poor header detection capabilities: by cutting too much into the lower part of the signal spectrum, "DM filtering" distorts the header waveform which is then not recognized. 


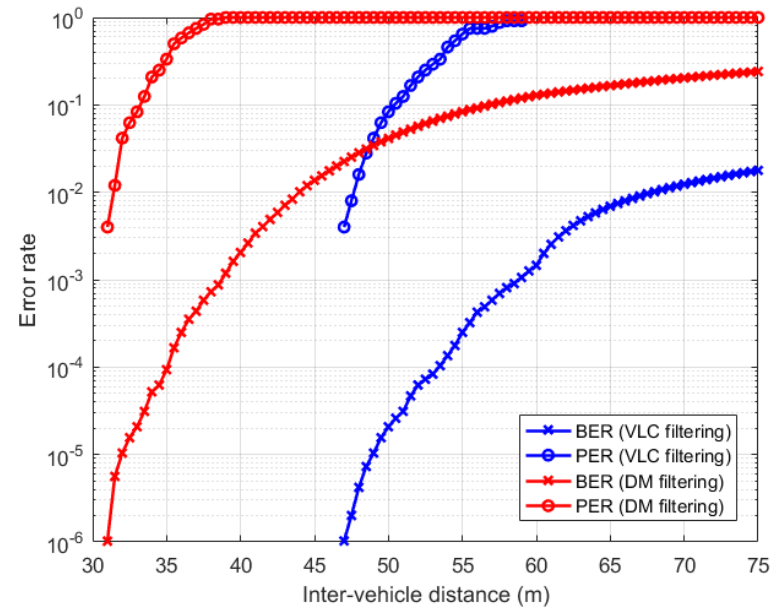

Fig. 10. BER and PER evolution against the $\mathrm{V} 2 \mathrm{~V}$ distance by steps of 50 cm with both "VLC filtering" and "DM filtering".

We can also note that the distribution of the errors is rather uniform, as the PER reaches quickly $100 \%$ which means each packet contains at least one error. More importantly, these results show the 'error-free' communication distance is in the best case around $45 \mathrm{~m}$. Therefore, the communication function of the VLCR is, from the longitudinal range point of view, perfectly suited for platooning use case, where the $\mathrm{V} 2 \mathrm{~V}$ distances are usually around $10 \mathrm{~m}$.

\section{E. Distance Measurement Performances}

The range-finding function is then evaluated. Fig. 11(a) shows the evolution of the distance measured with "DM filtering" and "VLC filtering" against the real V2V distance. These curves are obtained by varying the $\mathrm{V} 2 \mathrm{~V}$ distance from $1 \mathrm{~m}$ to $50 \mathrm{~m}$ and taking one measurement every $5 \mathrm{~cm}$. We can see first that both approaches provide a linear distance estimation from $1 \mathrm{~m}$ to $45 \mathrm{~m}$, even though local variations are clearly visible. After this distance, the measures obtained with "DM filtering" collapse whereas they keep increasing linearly with "VLC filtering". However, there is in both cases a non-negligible offset. This offset is due to the phase response of the reconstruction filters and to the recovery PLL which introduce additional delays resulting in an increase in the distance estimated.

By looking closer at these offsets, we can actually note that they are rather constant between $1 \mathrm{~m}$ and $45 \mathrm{~m}$. Therefore, in both cases, the distance provided can be corrected by removing their mean value. In practice, the mean offset can be estimated by addition of the delays introduced on both ends of the system. Each vehicle can periodically estimate its delay, for example by sending and processing an internal signal. The LV can then send the delay estimated through the VLC channel to the FV so that the latter can add it to its own delay and estimate the offset to remove.

After mean offset removal, we get a residual error which corresponds to the intrinsic error of the system. Fig. 11(b) shows the histogram of these errors in the case of "DM filtering", after correction over the range going from $1 \mathrm{~m}$ to $30 \mathrm{~m}$. It has a Gaussian-like distribution with zero mean and standard deviation $\sigma=0.33 \mathrm{~m}$ which means the system resolution, defined as the $\pm \sigma$ confidence interval, is around $66 \mathrm{~cm}$. Note that if correction is applied over different ranges, the distribution remains Gaussian-like. This behavior remains valid in the case of "VLC filtering" so the resulting standard deviations in both cases are given in Table II.

TABLE II

EVOLUTION, WITH THE DISTANCE, OF $\sigma_{\mathrm{DM}_{f}}$ AND $\sigma_{\mathrm{VLC}}$, THE STANDARD DEVIATIONS OF THE DISTANCE ERROR IN THE CASE OF "DM FILTERING" AND "VLC FILTERING".

\begin{tabular}{|c|c|c|}
\hline Correction range & $\sigma_{\mathbf{D M}_{f}}$ & $\sigma_{\mathbf{V L C}_{f}}$ \\
\hline 1 to $5 \mathrm{~m}$ & $24 \mathrm{~cm}$ & $29 \mathrm{~cm}$ \\
\hline 1 to $10 \mathrm{~m}$ & $26 \mathrm{~cm}$ & $32 \mathrm{~cm}$ \\
\hline 1 to $20 \mathrm{~m}$ & $33 \mathrm{~cm}$ & $40 \mathrm{~cm}$ \\
\hline 1 to $30 \mathrm{~m}$ & $33 \mathrm{~cm}$ & $43 \mathrm{~cm}$ \\
\hline 1 to $40 \mathrm{~m}$ & $40 \mathrm{~cm}$ & $44 \mathrm{~cm}$ \\
\hline 1 to $45 \mathrm{~m}$ & $44 \mathrm{~cm}$ & $47 \mathrm{~cm}$ \\
\hline
\end{tabular}

It appears clearly that, whatever the range of correction, "DM filtering" provides a better measurement resolution than "VLC filtering". This is due to the fact that "DM filtering" introduces less pulse distortions and thus enables a more accurate reconstruction of the signals received. This leads to a better stability of the PLL and, eventually, to a better resolution. However, the resolution in both cases gets worse with the range. This phenomenon can be explained by the fact that, as the V2V distance increases, the SNR of the signals received on both ends decrease. However, as illustrated by Fig. 9 , the similarity rate between the signals reconstructed and the original signals drops quickly with low SNR. Consequently, even though filtering helps recover rather clear signals, the distortions contained in these signals are getting larger with the distance, which impacts eventually the measurement error.

In addition, we can observe that the measurement error is periodic with a 1.33 cycles $/ \mathrm{m}$, and thus reaches a peak every $75 \mathrm{~cm}$, as shown by the fast Fourier transform (FFT) analysis on Fig. 11(c). This behavior is closely linked to the clock counting error $\delta M$ coupled with the slight phase variations of the reconstructed clock $s_{r}$ and the heterodyning process, as illustrated by Fig.5.

In any case, the overall measurement performances of the VLCR are worse than those of the VLR alone, which can provide distance resolutions under $30 \mathrm{~cm}$ over a range of 30 $\mathrm{m}$ [23]. This gap can be explained once again by Fig. 9: the reconstruction properties of the filters used by the VLR alone are way better than those of both the "DM filtering" and "VLC filtering" approaches. Consequently, even though the addition of the communication function is of great benefit, it comes necessarily with a degradation in the range-finding performances.

\section{Experimental Study of the RANGe-Finding AND COMMUNICATION FUNCTIONS}

Now that the VLCR has been described theoretically and validated through simulations, we can implement prototypes of the communication and range-finding functions in order 


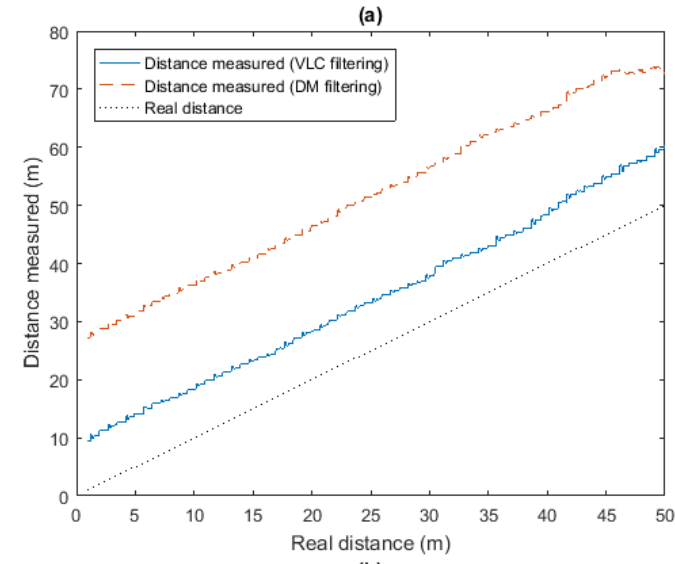

(b)

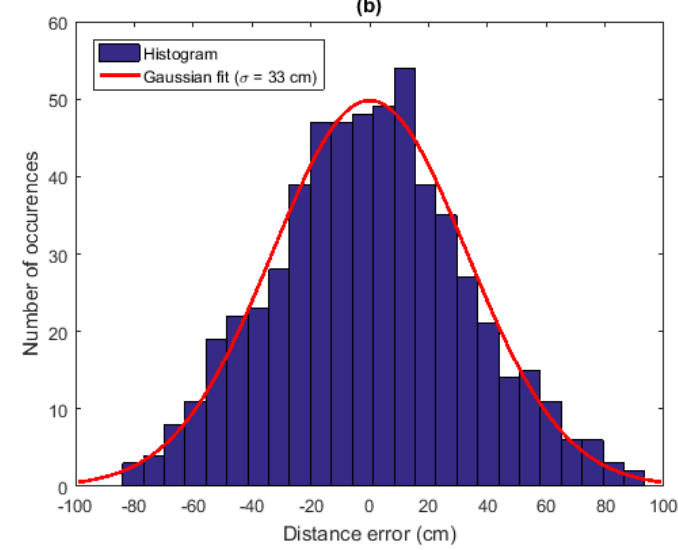

(c)

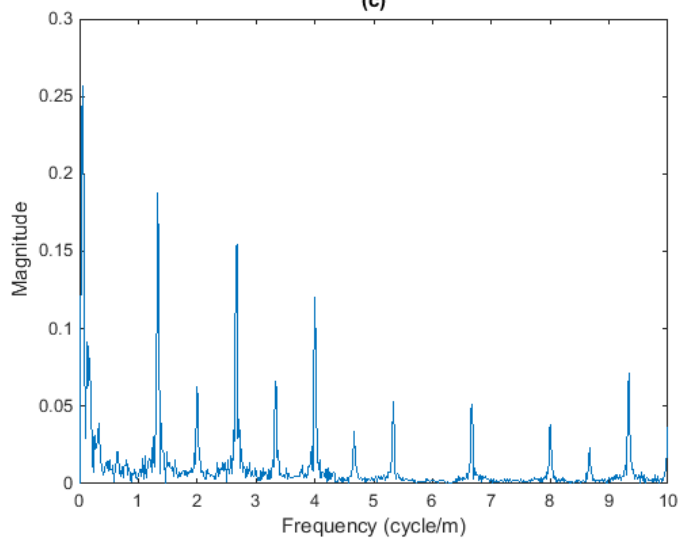

Fig. 11. (a) Evolution, against the real distance (black dotted line), of the estimated distance using "DM filtering" (red dashes) and "VLC filtering" (blue curve), (b) histogram of the error after correction over the range $1 \mathrm{~m}$ to 30 $\mathrm{m}$ in the case of "DM filtering" with its Gaussian fit curve and (c) FFT of this error.

to confirm they are functional in reality. The implementation of the prototypes is first described in Section V-A. Then, the range-finding function is demonstrated in Section V-B. Finally, the tests of the communication function are detailed in Section V-D. Consequently, note that the VLCR is not properly implemented here, as both functions are built and tested separately. This choice was made to focus on the validation of the range-finding function, which is the major novelty of our system.

\section{A. Prototypes Implementation}

1) Emitter Block: The signal emission, corresponding to the blocks 'LED' and 'Emitter' on Fig. 1, is made in practice using an LED light source controlled by an LED driver. The light source used on the FV side is a COTS white headlamp based on a Nichia NC4W121A high-power phosphor LED. The overall module is controlled with a forward current limited to 600 $\mathrm{mA}$, which gives a maximum luminous intensity of $16750 \mathrm{~cd}$ compliant with standard regulations for low beam headlamps [34]. On the LV side, another COTS white headlamp is used, but it is under-driven to meet taillights regulations [35].

Both light sources have a $3 \mathrm{~dB}$ bandwidth of $1.4 \mathrm{MHz}$, so the frequency of operation $f_{e}$ is, as previously, set to $1 \mathrm{MHz}$. In order to drive these lamps, a specific LED driver is designed and built. This driver converts the $1 \mathrm{MHz}$ small current complementary metal oxide semiconductor (CMOS) square signal of command $s_{e}$ or $s_{e^{\prime}}$, produced by a field programmable gate array (FPGA), into the $600 \mathrm{~mA}$ control signal. It is thus simply a metal oxide semiconductor FET (MOSFET), which gate is controlled by the signal of command, first amplified with an optocoupler so that the appropriate amount of current can flow through the LED.

2) Signal Reception: $P D$ and Processing Card: After their emission, the light signals are collected and processed by dedicated electronic cards in order to be reconstructed: this is the signal reception chain, represented on Fig. 2(b) and corresponding to the blocks 'PD' and 'Receiver' in Fig. 1. First, the light signal is collected with a PD which produces a photocurrent turned into a voltage and amplified by a TIA. These two blocks are implemented with a COTS photoreceiver Thorlabs PDA8A of bandwidth $50 \mathrm{MHz}$ and gain 50 $\mathrm{kV} / \mathrm{A}$.

When used in communication mode, this photo-receiver is directly connected to a Tektronix MDO3054 oscilloscope for offline decoding with MATLAB. This offline decoding is the same as the one implemented for the simulations and thus consists in "VLC filtering" and zero-crossing detection in order to retrieve the data stream transmitted and measure the error rate performances of the system.

On the other hand, when the system is used in rangefinding mode, the Thorlabs PDA8A is connected to a custommade reconstruction card embedding an 8-th order bandpass filter of center frequency $1 \mathrm{MHz}$ and bandwidth $100 \mathrm{kHz}$, amplification stages providing a maximum gain of $200 \mathrm{~V} / \mathrm{A}$ and a high-speed comparator. This card can turn noisy input signals of a few millivolts into a clean square CMOS signal of frequency $f_{e}$. This CMOS signal is then used to feed the LED driver in the case of the LV or an FPGA, for phase-shift measurement, in the case of the FV. As already detailed in [24], this card has very good reconstruction properties down to SNR as low as $5 \mathrm{~dB}$. However, the whole processing chain introduces a delay between the input and output signals that is around 933 ns with the card used on the LV and $928 \mathrm{~ns}$ with the card used on the FV. These values are, in addition, varying with the atmospheric conditions and especially with the temperature which will necessarily have an impact on the distance estimated, as we will see in Section V-D.

3) Phase-Shift Measurement Algorithm: Once the signal received from the $\mathrm{LV}$ is reconstructed into a stable square 
signal $s_{r}$, it is compared with the original square wave $s_{e}$ for phase-shift measurement. This phase-shift measurement step is performed digitally by an FPGA which has actually three main roles: generate the data stream $m_{e}$ and thus the clock signal $s_{e}$, heterodyne $s_{e}$ and $s_{r}$ and, finally, measure their phase-shift, which corresponds to the blocks 'Data', 'Message Encoding', 'Clock Generator' and 'Phase Measurement' in Fig. 1.

In practice, since the range-finding and communication functions are tested separately, the FPGA is only used as a Manchester data source operating at $500 \mathrm{kbps}$ in communication mode (no need of phase-shift measurement) whereas it only generates a square signal of frequency $f_{e}=1 \mathrm{MHz}$ in range-finding mode (no need of data and message encoding). In both case, the resulting signal is continuously fed to the LED driver. In addition, in range-finding mode, the PLL generates the counter clock $s_{\text {clock }}$ of frequency $f_{\text {clock }}=100$ $\mathrm{MHz}$ used for auto-digital phase measurement. Finally, a second PLL generates the heterodyning clock $s_{h}$ of frequency $f_{h}$.

This heterodyning clock is obtained using an heterodyning factor $r=3999$. As explained in Section III-C, such a value leads to an interesting compromise between measurement error and refresh rate. The resulting heterodyning frequency $f_{h}$ is in this case $999750 \mathrm{kHz}$. Unfortunately, the resolution of the internal PLL of the FPGA is not sufficient to support this exact frequency, which is measured instead at $999746.9 \mathrm{kHz}$ with a frequency counter Agilent 53132A. This frequency corresponds to a true heterodyning factor $r=3950.007$. This clock is then used to cadence a D flip-flop gate coded in hardware description language (HDL), as the rest of the autodigital phase measurement algorithm represented on Fig. 4. This algorithm outputs the number of clock ticks $M$ that is stored and then converted into phase-shifts and distances with MATLAB. Note that since no averaging is used $(\mathrm{N}=1)$, the refresh rate of the measurement (7) is $506 \mathrm{~Hz}$.

Tests of this algorithm show that the phase-shift measured is linear with slope +1 from 0 to $180^{\circ}$, and then linear again from $180^{\circ}$ to $360^{\circ}$ but with a slope -1 . This confirms that our method has a non-ambiguity range of $\pi$ in terms of phase-shift, which corresponds to (6) in terms of distance. In addition, it is found that the each measurement contains a small phaseshift error, and thus a distance error. As explained in Section III-B, these errors are due to the limited resolution of the heterodyning step. However, they remain under $3 \mathrm{~cm}$ which is in accordance with the maximum error (9) equal here to 3.79 $\mathrm{cm}$ when $r=3950.007$. This behavior confirms eventually that our phase-shift measurement algorithm is fully functional.

\section{B. Validation of the Range-Finding Function}

1) Experimental Set-Up and Protocol: The set-up used to validate the range-finding function is represented on Fig. 12. The FV and LV are both modeled by moving tables on which all the equipment is installed, and placed in a controlled laboratory environment. The table modeling the FV is fixed whereas the table representing the $\mathrm{LV}$ is moved in order to vary the $\mathrm{V} 2 \mathrm{~V}$ distance from $5 \mathrm{~m}$ to $25 \mathrm{~m}$ by steps of 50 $\mathrm{cm}$. Both tables are kept on the same longitudinal axis so that both vehicles can be considered aligned in a straight line. At each step, the 'true' distance is first measured with a Laserliner LaserRange-Master 40 rangefinder which can measure distances up to $30 \mathrm{~m}$ with a typical resolution of $\pm 2 \mathrm{~mm}$. Then, 4096 consecutive distance estimations are performed, in static conditions, with the system.

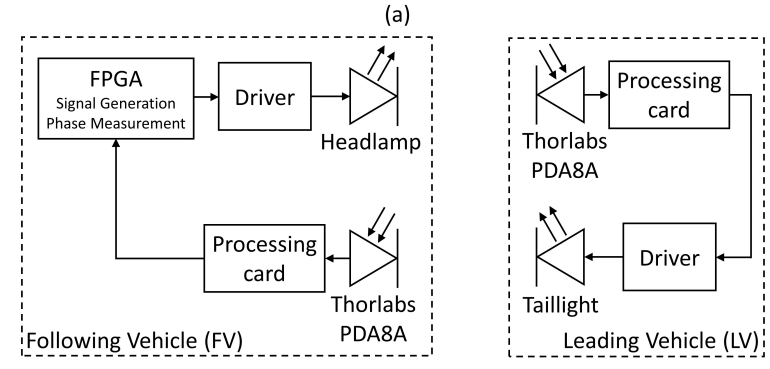

(b)

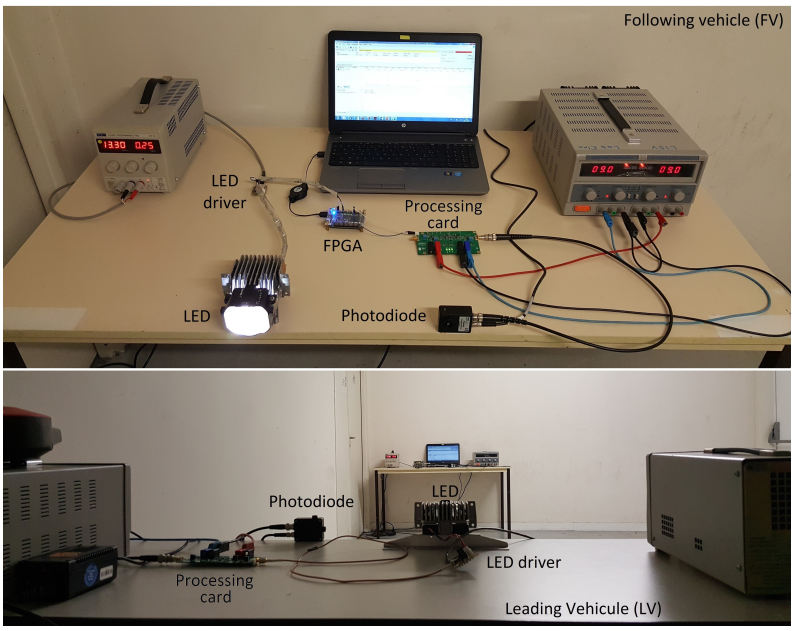

Fig. 12. (a) Structure of the set-up used during the range-finding experiments and (b) photo of this set-up with at the top the FV end and at the bottom the LV end.

2) General Behavior and Measurement Correction: Fig. 13 shows the evolution of the average distance measured against the real $\mathrm{V} 2 \mathrm{~V}$ distance. We can observe first that there is an inflection point around $10.5 \mathrm{~m}$. This behavior is induced by the processing delays added by the reconstruction cards used on both ends of the system. These delays are such that the overall phase-shift between $s_{e}$ and $s_{r}$ at $5 \mathrm{~m}$ is larger than $\pi$. Consequently, as the $\mathrm{V} 2 \mathrm{~V}$ distance and thus the return trip TOF increases, the overall phase-shift gets larger, which leads to a decrease in the value output by the system, in accordance with the observations made in Section V-A3. Then, at $10.5 \mathrm{~m}$, the sum of the various phase-shifts is such that $s_{e}$ and $s_{r}$ are in phase, which leads to a distance measured null. After this value, the phase-shift is again positive, but this time below $\pi$, hence the rising section on Fig. 13.

Fortunately, the processing delays remain, as in the simulations, rather constant. We can indeed observe from the linear fits on Fig. 13 that both the decreasing and rising sections are rather linear, with an absolute slope close to unity. Consequently, it should be possible to apply a correction rule on these first results to remove the electronic delays and thus the inflection point with the corresponding measurement 


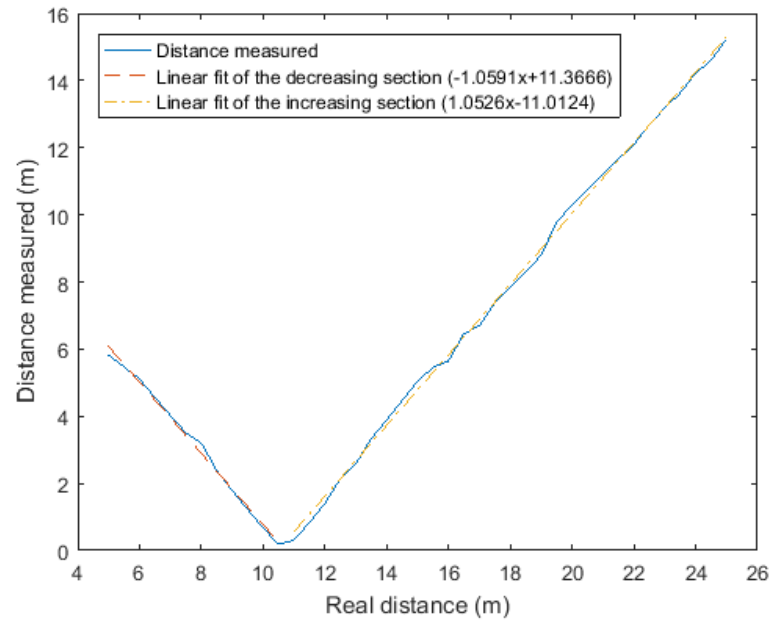

Fig. 13. Evolution of the average distance measured by the prototype against the real distance with the linear fits of its decreasing and increasing sections.

error. In practice, the measures are corrected by first, adding a controlled delay so that the final phase-shifts remain between 0 and $\pi$, whatever the true $\mathrm{V} 2 \mathrm{~V}$ distance. Then, a linear fit of the averaged distance measurement is performed in order to define the offset that is finally removed. Note that such a correction assumes the various delays remain constant with time, which is here relevant given the duration of the whole experiment. However, in real conditions, this correction step will have to be repeated periodically because, for example, of the temperature variations the vehicle can experience throughout a journey. In such a case, auto-calibration method for real conditions functioning can be implemented, as the one quickly proposed in Section IV-E.

\section{Measurement Error and Resolution}

The fixed correction rule previously exposed allows to remove the inflection point observed on Fig. 13 and thus a large part of the initial measurement error, defined as the difference between the distances output by the system and the true distances. However, there is still after correction a residual error. To characterize this error, we can study the distributions of the 4096 consecutive measures taken at each distance tested. It is found that this distribution is Gaussianlike with, each time, a specific standard deviation $\sigma$ and mean value. First, it must be noted that the correction rule does not change this shape but simply shifts the mean value closer to zero. For example, the mean distance measured for a true distance of $18 \mathrm{~m}$ is $7.8 \mathrm{~m}$. After correction, this value reaches $17.95 \mathrm{~m}$, which gives a mean error after correction of $5 \mathrm{~cm}$. More generally, these mean errors are varying between $-35 \mathrm{~cm}$ and $35 \mathrm{~cm}$, without specific distribution, but $75 \%$ of them are actually between $-16 \mathrm{~cm}$ and $16 \mathrm{~cm}$.

Then, the Gaussian like distribution of the consecutive measures made for a same true distance allow us to determine, for each distance tested, a distance measurement resolution. This resolutions is, as in the simulations, defined as twice the standard deviation $\sigma$ of the measurements distribution for each distance. Such a definition means that $70 \%$ of the values output for a specific distance by the system will be contained in the interval centered on the mean value after correction at this distance plus or minus one standard deviation $\sigma$ at this distance. Table III lists the resolution measured for the various true distances tests, as well as the corresponding percentage of error.

TABLE III

Distance RESOlUtion AGAINST THE TRUE V2V Distance.

\begin{tabular}{|c|c|c|}
\hline True distance (m) & Resolution $2 \sigma(\mathbf{c m})$ & \% of error \\
\hline 4.988 & 14.18 & 2.8 \\
\hline 7.483 & 16.44 & 2.2 \\
\hline 10.001 & 23.60 & 2.4 \\
\hline 12.49 & 24.52 & 2.0 \\
\hline 15.001 & 30.94 & 2.1 \\
\hline 17.498 & 31.68 & 1.8 \\
\hline 20.027 & 52.16 & 2.6 \\
\hline 22.499 & 60.90 & 2.7 \\
\hline 24.985 & 77.04 & 3.1 \\
\hline
\end{tabular}

These variations can be explained by the phase noise of the signal reconstructed $s_{r}$, and thus by the limits of the hardware implementation of our prototype. $s_{r}$ is indeed supposed to be a square signal of fixed frequency $f_{e}$ and duty cycle $50 \%$. However, the reconstruction process, mixed with the limited bandwidth of the emitters on both sides, cannot guarantee such a stability in frequency and duty cycle. The resulting fluctuations will necessarily vary the phase-shift between $s_{r}$ and the signal first sent $s_{e}$, and eventually the distance measured. Given the fact that this distribution is Gaussianlike, we can deduce this phase noise is Gaussian and that it gets stronger with the V2V distance, that is as the SNR of the signals received on both ends of the system decreases.

These various results finally allow us to plot, on Fig. 14, the evolution of the distance measured by our range-finding prototype, after correction, and with the corresponding error bars. We can conclude from these results that our system is functional, even though the performances reached so far are still lower than the measurement resolution of $30 \mathrm{~cm}$ at $30 \mathrm{~m}$ reported in [23]. This difference in performance can mainly be explained by the phase noise of $s_{r}$ caused by the various distortions induced by the limited bandwidth of the transmit LED and of the PD, or by the filtering and comparison stages used to reconstruct $s_{r}$.

\section{Validation of the Communication Function}

Finally, the communication function of the VLCR is evaluated. The experimental set-up used for these experiments is represented on Fig. 15 and is very similar to the set-up of Fig. 12 , used to validate the range-finding function. On one end of the system, the FPGA generates the Manchester data stream at a data rate $R_{b}=500 \mathrm{kbps}$. This data streams is fed to the LED driver, which controls the light intensity of the LED. On the other end of the system, the Thorlabs PDA8A photo-receiver collects the lights signal and turns it into a voltage signal that is directly sampled by a Tektronix MDO3054 oscilloscope. The resulting samples are then processed offline using MATLAB to evaluate the error rate performances of the link. 


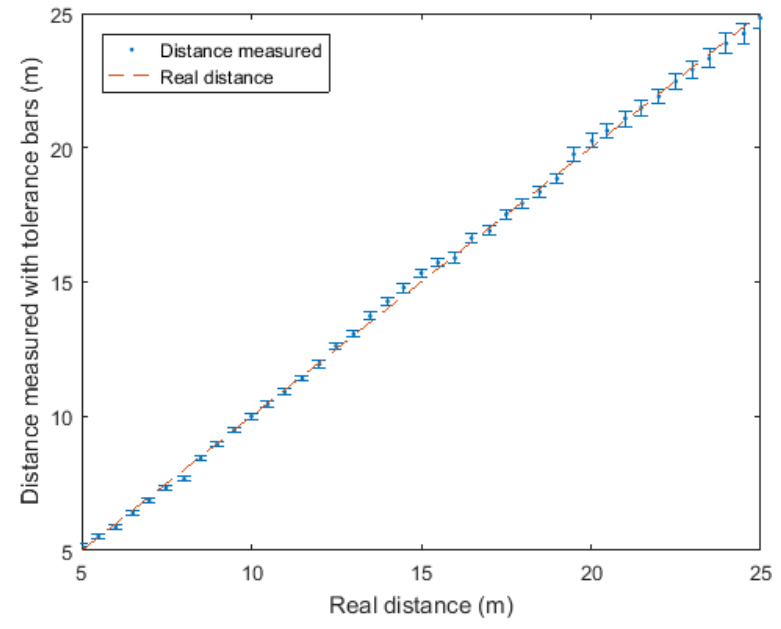

Fig. 14. Evolution of the distance measured after correction with the corresponding error bars (blue dots) and of the true V2V distance (red dashes).
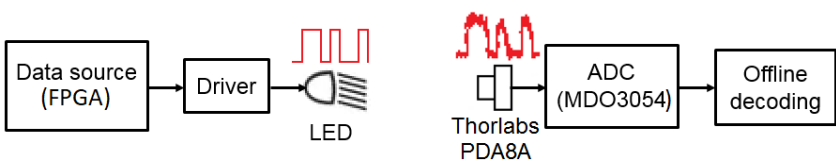

Fig. 15. Experimental set-up used to test the communication function of the VLCR.

As in Fig. 12(b), both vehicles are modeled by tables that can be moved to simulate the V2V distance. These tables are placed in an indoor corridor of length $30 \mathrm{~m}$, illuminated with standard neon tubes driven at $50 \mathrm{~Hz}$ providing a constant average illuminance of 150 lux. The V2V distance $d$ is then varied from $5 \mathrm{~m}$ to $30 \mathrm{~m}$ by steps of $5 \mathrm{~m}$ and at each step, $10^{6}$ data bits divided into packets of 4000 bits and separated by a header $\mathrm{H}=00001111$ are sent.

The MATLAB post-processing consists, as already mentioned, in a "VLC filtering" stage followed by zero-crossing detection so that the signal reconstructed can be compared to the data signal for BER and PER evaluation. After this offline processing stage, there were actually no errors found in the reconstructed data signal. Therefore, we can conclude, as summed-up in Table IV, that the BER remains below $10^{-6}$ up to at least $30 \mathrm{~m}$. Note that such a result was obtained using the LED light in both the low-beam headlamp mode and the taillight mode. In addition, the same results were obtained at a data rate of $1 \mathrm{Mbps}$. In any case, we can conclude from these experiments that the communication function of the VLCR, which is nothing more than a simple VLC system, is suited for platooning applications, at least in terms of operation range.

TABLE IV

EVOLUTION OF THE BER With THE TRUE V2V DisTANCE AT 500 KBPS.

\begin{tabular}{|c|c|}
\hline V2V distance (m) & BER \\
\hline 30 & $<10^{-6}$ \\
\hline
\end{tabular}

\section{CONCLUSIONS AND Future WORKS}

\section{A. Summary of the Findings}

In this paper, a new concept of communication and rangefinding system called VLCR and based on the automotive headlamps and taillights is proposed for the first time in the literature. The VLCR is a simple VLC system over which is added a distance measurement layer, performed through the measurement of the phase-shift between clock signals contained in the data signals. The theoretical basis of this system are detailed and an analytical study of the sources of errors is carried out. A simulation study then validates the operation principles of the VLCR. Finally, both its communication and range-finding functions are validated experimentally in order to bring a proof of concept.

Although not optimized from the hardware point of view, this prototype enables distance measurement up to $25 \mathrm{~m}$ and ensures a measurement resolution of around $24 \mathrm{~cm}$ at 10 $\mathrm{m}$, with a refresh rate of $506 \mathrm{~Hz}$. In parallel, it enables data transmission at $500 \mathrm{kbps}$ of 4000 bits packets over at least $30 \mathrm{~m}$ with a BER below $10^{-6}$. The various simulation and practical studies also show that the VLCR does not sacrifice any bandwidth to support the range-finding function since this function is performed in parallel. On the other hand, the range-finding performances are degraded by the communication function because of the inexact reconstruction of the data signal and the resulting phase variations of the clock reconstructed by the clock recovery PLL. Simulations show that the distance measurement resolution is twice larger with the VLCR than with the VLR only.

As a reminder, highway platooning requires a nominal $\mathrm{V} 2 \mathrm{~V}$ distance of 8 to $10 \mathrm{~m}$, a refresh rate of the distance measurement of at least $50 \mathrm{~Hz}$, a measurement error of a few centimeters, and a communication function ensuring a $20 \mathrm{~ms}$ latency when transmitting small packets of 400 bits. These results thus show that the automotive lighting can be used as a complement to lidar, radar and radio communication systems in a simple a low cost manner. Except for the expensive offthe-shelf photo-receivers and FPGA development board, the bill of material for the rest of the components is indeed around $\$ 15$. Therefore, we can easily imagine that an optimized version of the VLCR with dedicated components would be very cost-competitive.

\section{B. Discussion and Future Works}

Despite promising results, the distance measurement performances of our prototype must be optimized to reach standard platooning requirements. The main way to increase these performances is to enhance the hardware implementation of the prototype. For example, focusing lenses carefully designed could be used to optimize the amount of light detected by a front-end also improved in terms of gain and noise immunity. From the reconstruction step point of view, a better hardware design combined with a meticulous choice of the components would probably enhance the stability of the reconstructed signals. The resulting prototype would then be tested first under various lighting and interferences scenario and then in real traffic conditions to deepen the performance analysis. 
Finally, the communication function would be added according to the principles proposed and validated through simulations in this paper, and then tested in real networked conditions.

In parallel, other solutions will be investigated. Our work has been focused so far on implementing our system according to the framework defined by the VLC standard IEEE 802.15.72011 [30], which recommends to use pulse modulations. However, joint communication and distance measurement has been a hot topic lately, especially with advanced modulation schemes like orthogonal frequency division multiplexing (OFDM). For example, in the IEEE 802.11-2016 standard, stations can measure their inter-distance using the fine time measurement (FTM) technique based on FTM frames containing timestamps sent back and forth to measure a timestamp difference corresponding to the TOF. Such a method could actually be used with the current version of the VLCR since only the data frames must be changed. It would then add a second distance measurement after the one given by phase-shift measurement, but at the cost of the bandwidth dedicated to V2V communication. This bandwidth could thus be increased using OFDM, but in this case, the clock recovery PLL, on which the distance measurement is based, would not work anymore. Consequently, moving toward OFDM to reach high data rates would require to change the range-finding method and use, for example, pilot symbols based techniques as proposed in [37].

\section{REFERENCES}

[1] C. Bergenhem, S. Shladover, E. Coelingh, C. Englund, and S. Tsugawa, "Overview of platooning systems," presented at the 19th ITS World Congress, Vienna, Austria, 22-26 Oct. 2012.

[2] S. Tsugawa, S. Jeschke, and S.E. Shladover, "A Review of Truck Platooning Projects for Energy Savings,” IEEE Trans. Intell. Veh. 1(1), 68-77 (2016)

[3] A. Geiger, M. Lauer, F. Moosmann, B. Ranft, H. Rapp, C. Stiller, and J. Ziegler, "Team AnnieWAY's Entry to the 2011 Grand Cooperative Driving Challenge," IEEE Trans. Intell. Transp. Syst., vol. 13, no. 3, pp. 1008-1017, Sep. 2012.

[4] P. S. Jootel, "SAfe Road TRains for the Environment - Final Report," http://www.sartre-project.eu/en/publications/Sidor/default.aspx.

[5] European Truck Platooning Network, "Vision truck platooning 2025", 2016. [Online] Available: https://www.eutruckplatooning.com/Support/ Videos/Vision+Truck+Platooning+2025+TNO/default.aspx

[6] S. Al-Sultan, M. Al-Doori, and H. Zedan, "A comprehensive survey on vehicular Ad Hoc network," J. Netw. Comput. Appl., vol. 37, pp. 380-392, Jan. 2014.

[7] A. Festag, "Cooperative intelligent transport systems standards in europe," IEEE Commun. Mag., vol. 52, no. 12, pp. 166-172, Dec. 2014.

[8] "Specification of Cooperative Awareness Basic Service," ETSI EN 302 687-2 (V1.3.1), Sep. 2014.

[9] A. Böhm, M. Jonsson, and E. Uhlemann, "Performance comparison of a platooning application using the IEEE 802.11p MAC on the control channel and a centralized MAC on a service channel," in Proc. 9th IEEE WiMob, 2013, pp. 545-552.

[10] M. Y. Abualhoul, M. Marouf, O. Shagdar, and F. Nashashibi, "Platooning control using visible light communications: A feasibility study," in Proc. 16th IEEE ITSC, 2013, pp. 1535-1540.

[11] S. Ishihara, R. V. Rabsatt, and M. Gerla, "Improving reliability of platooning control messages using radio and visible light hybrid communication," in Proc. IEEE VNC, 2015, pp. 96-103.

[12] M. Segata, R. L. Cigno, H.-M. Tsai, and F. Dressler, "On platooning control using IEEE 802.11p in conjunction with visible light communications," in Proc. IEEE/IFIP WONS, 2016, pp. 14.

[13] M. Akanegawa, Y. Tanaka, and M. Nakagawa, "Basic study on traffic information system using LED traffic lights," IEEE Trans. Intell. Transp. Syst., vol. 2, no. 4, pp. 197-203, Dec. 2001.
[14] I. Takai, T. Harada, M. Andoh, K. Yasutomi, K. Kagawa, and S. Kawahito, "Optical Vehicle-to-Vehicle Communication System Using LED Transmitter and Camera Receiver," IEEE Photonics J., vol. 6, no. 5, pp. 1-14, Oct. 2014.

[15] S.-H. Yu, O. Shih, H.-M. Tsai, N. Wisitpongphan, and R. Roberts, "Smart automotive lighting for vehicle safety," IEEE Commun. Mag., vol. 51, no. 12, pp. 50-59, Dec. 2013.

[16] A.-M. Cailean, B. Cagneau, L. Chassagne, M. Dimian, and V. Popa, "Novel Receiver Sensor for Visible Light Communications in Automotive Applications," IEEE Sens. J., vol. 15, no. 8, pp. 4632-4639, Aug. 2015.

[17] J. Wenger, "Automotive radar - status and perspectives," in Proc. IEEE CSIC'05, 2005, pp. 21-24.

[18] J. Hasch, "Driving towards 2020: Automotive Radar technology trends," in Proc. IEEE ICMIM, 2015, pp. 1-4.

[19] J. Lee, Y.-A. Li, M.-H. Hung, and S.-J. Huang, "A Fully-Integrated 77GHz FMCW Radar Transceiver in 65-nm CMOS Technology," IEEE J. Solid-State Circuits, vol. 45, no. 12, pp. 2746-2756, Dec. 2010.

[20] C. Niclass, M. Soga, H. Matsubara, M. Ogawa, and M. Kagami, "A 0.18-um CMOS SoC for a 100-m-Range 10-Frame/s 200 96-Pixel Timeof-Flight Depth Sensor," IEEE J. Solid-State Circuits, vol. 49, no. 1, pp. 315-330, Jan. 2014

[21] M. Goppelt, H.-L. Blöcher, and W. Menzel, "Automotive radar - investigation of mutual interference mechanisms," Adv. Radio Sci., vol. 8, pp. 55-60, Sep. 2010

[22] G. Kim, J. Eom, and Y. Park, "An Experiment of Mutual Interference between Automotive LIDAR Scanners," in Proc. 12th ITNG, 2015, pp. 680-685.

[23] B. Béchadergue, L. Chassagne, and H. Guan, "Visible light phase-shift rangefinder for platooning applications," in Proc. 19th IEEE ITSC, 2016, pp. $2462-2468$.

[24] B. Béchadergue, L. Chassagne, et H. Guan, "Vehicle-to-Vehicle Visible Light Phase-Shift Rangefinder Based on the Automotive Lighting", IEEE Sens. J., vol. 18, no 13, pp. 5334-5342, July 2018.

[25] K. Mizui, M. Uchida, and M. Nakagawa, "Vehicle-to-vehicle communication and ranging system using spread spectrum technique (Proposal of Boomerang Transmission System)," in Proc. 43rd IEEE VTC, 1993, pp. $335-338$.

[26] A. J. Suzuki and K. Mizui, "Laser radar and visible light in a bidirectional V2V communication and ranging system," in Proc. IEEE ICVES'15, 2015, pp. 19-24.

[27] Z. Li, L. Liao, A. Wang, and G. Chen, "Vehicular optical ranging and communication system," EURASIP J. Wirel. Commun. Netw., vol. 2015, no. 1, pp. 1-6, Jul. 2015.

[28] C.-Y. Wen, R. D. Morris, and W. A. Sethares, "Distance Estimation Using Bidirectional Communications Without Synchronous Clocking," IEEE Trans. Signal Process., vol. 55, no. 5, pp. 1927-1939, May 2007.

[29] S. Liu, J. Tan, and B. Hou, "Multicycle synchronous digital phase measurement used to further improve phase-shift laser range finding," Meas. Sci. Technol., vol. 18, no. 6, pp. 1756-1762, Jun. 2007.

[30] S. Rajagopal, R. D. Roberts, and S.-K. Lim, "IEEE 802.15.7 visible light communication: modulation schemes and dimming support," IEEE Commun. Mag., vol. 50, no. 3, pp. 72-82, Mar. 2012.

[31] I. Glover and P. M. Grant, Digital Communications, 3rd ed. Pearson Education, 2010.

[32] J. M. Kahn and J. R. Barry, "Wireless infrared communications," Proc. IEEE, vol. 85, no. 2, pp. 265-298, Feb. 1997.

[33] A. J. C. Moreira, R. T. Valadas, and A. M. de O. Duarte, "Optical interference produced by artificial light," Wirel. Netw., vol. 3, no. 2, pp. 131-140, May 1997.

[34] "Headlamps emitting an asymmetrical passing-beam," ECE Regulation No. 112 - Rev.3, Jan. 2013.

[35] "Position, stop and end-outline lamps," ECE Regulation No. 7 - Rev.6, Nov. 2012.

[36] "IEEE Standard for Information technology-Telecommunications and information exchange between systems Local and metropolitan area networks-Specific requirements - Part 11: Wireless LAN Medium Access Control (MAC) and Physical Layer (PHY) Specifications," in IEEE Std 802.11-2016 (Revision of IEEE Std 802.11-2012), vol., no., pp.1-3534, 14 Dec. 2016.

[37] C. D. Ozkaptan, E. Ekici, O. Altintas, and Chang-Heng Wang, "OFDM Pilot-Based Radar for Joint Vehicular Communication and Radar Systems", in 2018 IEEE VNC, Dec. 2018. 


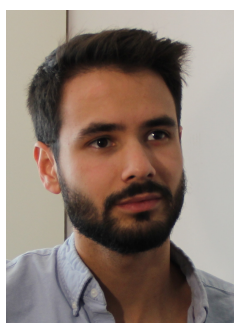

Bastien Béchadergue received the engineer degree from ISAE-Supaero, Toulouse, France and the M.S. in communication and signal processing from Imperial College London, London, UK in 2014. $\mathrm{He}$ received then his Ph.D. degree in signal and image processing in 2017 from the UVSQ, University Paris-Saclay, for his work on visible light communication and range-finding for automotive applications. His current research interests include optical wireless communications including LiFi but also optical camera communications.

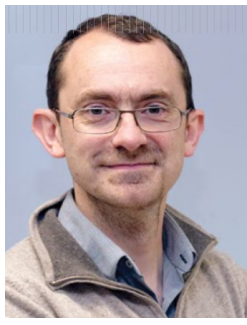

Luc Chassagne received a B.S. in Electrical Engineering (1994) from Supelec (France) and received his Ph.D. (2000) in optoelectronics from the University of Paris XI, Orsay (France) for his work in the field of atomic frequency standard metrology. $\mathrm{He}$ is now Professor and Director of the LISV laboratory at the University of Versailles. The topics of interest in his research are nanometrology, sensors and Visible Light Communications.

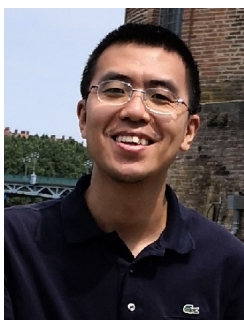

Hongyu Guan received a B.S. in Electrical Engineering (2007) from ENSEIRB (France) and his Ph.D. (2012) in computer science from the University of Bordeaux I, Bordeaux (France) for his work on embedded systems for home automation. He is now Research associate and chief project at the LISV laboratory, University of Versailles. His current research interests are Visible Light Communications, ubiquitous, data fusion, sensors and nanometrology. 